\title{
Agôn
}

Revue des arts de la scène

HS 1 | 2011

Mettre en scène l'événement

\section{Recopiage et imitation}

Examen des sources de 11 septembre 2001 de Michel Vinaver

\section{Simon Chemama}

\section{(2) OpenEdition}

Journals

Édition électronique

URL : http://journals.openedition.org/agon/1810

DOI : 10.4000/agon.1810

ISSN : 1961-8581

Éditeur

Association Agôn

Référence électronique

Simon Chemama, "Recopiage et imitation », Agôn [En ligne], HS 1 | 2011, mis en ligne le 05 septembre 2011, consulté le 17 avril 2020. URL : http://journals.openedition.org/agon/1810 ; DOI : https://doi.org/10.4000/agon.1810 


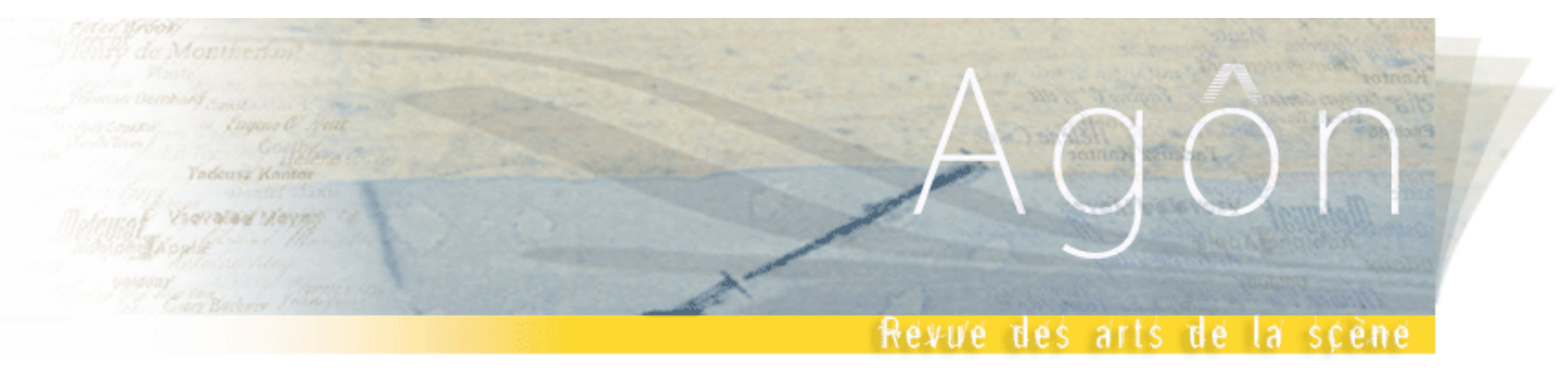

\title{
Recopiage et imitation \\ Examen des sources de 11 septembre 2001 de Michel Vinaver
}

\author{
Simon CHEMAMA
}

\author{
Quelqu'un qui s'exprime par proverbes est un archaïque, \\ par citations un cuistre, par citations sans mentionner \\ leur auteur (comme fait Jean-Luc Godard) un postmoderne. \\ (in Petit inventaire des citations malmenées, \\ Paul Desalmand et Yves Stalloni, Albin Michel, 2009)
}

11 Septembre 2001 de Michel Vinaver a eu une répercussion importante sur le monde du théâtre (français mais aussi, dans une moindre mesure, étranger), ne serait-ce qu'en nombre de traductions, lectures et mises en scène. Ce texte peut être vu comme le prolongement naturel des pièces précédentes de l'auteur, et leur aboutissement. Mais sur certains points, il tranche fortement avec le reste de son corpus. Nous allons nous intéresser ici aux sources de la pièce et à la façon dont Michel Vinaver s'en est servi. Nous analyserons le choix des passages prélevés dans les journaux, leur transformation et repèrerons les rares ajouts. L'enjeu n'est pas de démonter le texte, de le disséquer froidement pour en exposer les entrailles, et encore moins de dénoncer des "plagiats »; mais au contraire d'en révéler la singulière littérarité. Une littérarité qui aide même, affirmerons-nous, à penser la littérature. C'est en examinant le principe générateur de la pièce, que l'on pourra voir comment fonctionne la transfiguration opérée par Vinaver (pour évoquer l'ouvrage d'Arthur Danto, La Transfiguration du banal (1981), dont cet article est aussi une lecture $)^{1}$. Et nous nous demanderons, presque naïvement, ce que recouvre ce verbe «imiter» très employé par l'auteur à propos de la rédaction de 11 septembre 2001. Qu'est-ce qu'une imitation?

Michel Vinaver a 19 ans. Il rencontre Camus dans un grand hôtel new-yorkais, et lui dit «Je veux écrire », avant d'ajouter «mais tout a été écrit». Camus lui répond que non, et les deux hommes deviennent amis ${ }^{2}$. Cinquante-cinq ans plus tard, Vinaver pense peut-être toujours

\footnotetext{
${ }^{1}$ C'est aussi l'ambition du livre d'Antoine Compagnon, La Seconde Main (Éditions du Seuil, 1979) : la citation «sert à éprouver la valeur de la conversion que le livre opère du déjà dit, par la répétition et par l'entreglose » (p. 12, nous soulignons).

2 Olivier Todd, Albert Camus, une vie, 1996, p. 560. Si l'on veut creuser dans cette direction biographique, on peut rapporter aussi qu'à la lecture de « The Joker », l'un des premiers textes de l'auteur (publié en 1944 dans The Wesleyan Cardinal), le père de Michel Vinaver fut certain que son fils avait recopié un texte existant. Il l'accusait plus ou moins de plagiat, et cela à propos d'un texte qui était hautement personnel et fantastique...
} 
que « tout a été écrit », mais il dépasse la contradiction : à présent il s'en réjouit, puisque cela permet de « faire » une pièce.

\section{Génétique - l'imitation comme recopiage, tri et recomposition}

Nous ne pensons pas révéler un secret: Michel Vinaver a écrit explicitement que les mots de sa pièce sont «taken from daily newspapers $»^{3}$. Beaucoup de chercheurs ou de simples amateurs, curieux, ont sans doute tenté de vérifier cette indication, au moins sur une partie du texte. Un article de Catherine Douzou, par exemple, est tourné dans cette direction ${ }^{4}$. Personne, toutefois, n'a rendu compte systématiquement de l'origine du texte de Michel Vinaver. Plus dommageable : tout se passe comme si cette pièce désemparait les chercheurs. La plupart des articles qui y sont consacrés ne dépasse pas le simple constat, comme s'il fallait reproduire dans la critique ce qui constitue, chez Vinaver, un principe d'écriture dramatique.

Le travail que nous entreprenons a été initié par Fabien Spillmann (conseiller éditorial du projet «D'un 11 septembre à l'autre »), qui a fait le relevé exhaustif des sources de ce texte. Il semble que toutes les répliques (à l'exception de la dernière) proviennent de l'International Herald Tribune ${ }^{5}$. On comprend dès lors que l'un des enjeux sera de réfléchir sur cette notion de sameness of spelling (ou d'identité linguistique) que Gérard Genette, avec Nelson Goodman et contre Arthur Danto, définit comme étant peut-être la seule propriété constitutive d'une œuvre littéraire ${ }^{6}$.

Notons pour commencer que les différences minimes qu'on repère sur les noms de deux personnages de la pièce sont des erreurs de recopiage, non voulues ${ }^{7}$. Todd Beamer devient, dans la pièce, Todd Beaver, et Nat Alcamo, Nat Alamo ${ }^{8}$. Ces coquilles, on est toujours tenté de les lire comme des actes manqués. Comme tous les lapsus, elles ouvrent le sens, et nous apparaissent aussi comme des marqueurs de littérarité - justement parce qu'elles distinguent ce texte d'un rapport exact, d'un travail d'historien, d'une preuve. Alamo est plus suggestif qu'Alcamo : on pense au siège de Fort-Alamo, un des derniers épisodes de lutte contre le Mexique avant l'indépendance du Texas (Davy Crockett était dans le fort), ou même à A.L.A.M.O. (Atelier de Littérature Assistée par les Mathématiques et les Ordinateurs créé par Braffort et Roubaud en 1981). Quant au nom Beaver, il condense un faisceau de significations merveilleux. Le nom, tout d'abord, rappelait peut-être à l'auteur son personnage de Susan Beaver, la maîtresse de Jack dans L'Ordinaire (1981), qui, elle aussi, est victime d'un accident d'avion (dans les Andes, avec les dirigeants de la compagnie Housies). Les quatre dernières lettres [-aver], que Beaver et Vinaver

311 September 2001, «Foreword», in Théâtre complet 8, L'Arche, 2003, p. 132. La traduction française de la « Note liminaire» (puisque cette pièce est entièrement bilingue, anglais et français face à face), donne : «des paroles rapportées, provenant de la lecture de la presse quotidienne ». Nous verrons que cette traduction est signifiante.

4 Catherine Douzou, " 11 septembre 2001 de Vinaver. Babel implosée », in F. Fix et Fr. ToudoireSurlapierre (éd.), La citation dans le théâtre contemporain (1970-2000), Éditions universitaires de Dijon, coll. « Écriture », 2010, p. 93-102.

5 Articles parus entre le 9 septembre et le 9 octobre 2001 - ce journal est une édition internationale qui reprend, principalement, des articles du New York Times et du Washington Post; mais on trouve aussi dans le relevé de Spillmann un article provenant du Los Angeles Times du 20 septembre.

${ }^{6}$ Gérard Genette, L'œuvre de l'art, 1, Seuil, 1994, p. 88-89.

7 Sur un document de 2005, Vinaver (qui vient de lire le livre de Jim Dwyer et Kevin Flynn, 102 minutes. The Untold Story of the Fight to Survive inside the Twin Towers [Account based on 352 people's stories of whom 126 did not survive], Times Books, New York, 2005. ...) relève ces erreurs. Dans la plupart des mises en scène, le nom de Beamer est restitué correctement.

${ }^{8}$ Coquilles qui n'ont été repérées ni dans l'édition de la pièce seule, ni pour le Théâtre complet. 
partagent, font peut-être également de ce nom une signature (ou l'équivalent d'un autoportrait du peintre dans les représentations de foule de la Renaissance italienne). Enfin, l'expression to beaver away at something, qui signifie "travailler d'arrache-pied à quelque chose», serait presque une image du travail d'écriture. La dimension littéraire de ces deux lapsus semble presque évidente.

\title{
Classement des différents procédés
}

Essayons de classer les techniques d'écriture en fonction de l'intervention de l'auteur. Nous partirons des cas où la présence de celui-ci est la moins sensible (pure copie) pour aboutir au cas où elle est totale (pure invention). Dans l'idéal, cet exercice aura l'avantage de nous montrer sa propre insuffisance pour la compréhension de ce qu'est le travail littéraire?

La plupart des citations sont reproduites sans modification (autre que le fait d'être des citations) - ce que l'on peut appeler une technique de copier-coller. Les discours officiels (Bush, Rumsfeld, Ben Laden), ainsi que le feuillet d'instructions aux terroristes et le testament d'Atta sont fidèlement cités. Ailleurs on trouve des modifications élémentaires qui sont dues à la réintroduction du discours direct (passage du passé au présent notamment, changement de pronoms...). Par exemple cette réplique de Madeline :

\author{
MADELINE \\ Michael this plane \\ Has been hijacked \\ They have just gained access to the cockpit \\ The plane's now reversed direction \\ It's begun to descend rapidly (p. 138)
}

provient de cet extrait d'article :

«This plane has been hijacked,» Sweeney said, according to the FBI report. [...] But even as she was relating details about the hijackers, the men were storming the front of the plane and «had just gained access to the cockpit.» Then, she told Woodward, the plane suddenly changed direction and began to descend rapidly. ${ }^{10}$

Les propos cités directement par le journaliste sont repris tels quels, et ceux qui sont rapportés au discours indirect font l'objet d'une mise au net: passage du passé au présent, adaptation des marqueurs temporels à la situation d'énonciation (de « suddenly » à «now » - le « then », lui, ne peut logiquement avoir d'équivalent); quant à la proposition "was relating details », c'est ce qu'on appelle du discours narrativisé, dont Vinaver ne se permet pas d'inventer l'origine. Dans son ensemble, la séquence consacrée au journaliste et aux rescapés fonctionnent sur ces principes simples.

Mais nous voyons bien que nous ne rendons pas compte d'un élément pourtant essentiel : il s'agit du choix. Au sein d'un journal (et de l'ensemble des numéros d'un journal sur une période donnée), qu'est-ce qui détermine le choix de tel ou tel article ? Est-il possible de répondre à cette question? Peut-être (et nous proposerons, plus loin, une interprétation basée sur l'abandon de deux articles que Vinaver avait pourtant commencé à traiter), mais cela

9 Nous utilisons «littéraire» dans un sens large, qui n'exclut pas du tout la dimension plus spécifiquement « dramatique ».

${ }^{10}$ Eric Lichtblau, « After the attack. Desperate flight. Aboard Flight 11, a Chilling Voice », Los Angeles Times, 20 septembre 2001. Avec des extraits du FBI report investigation, interview of Michael Woodward, sept. 13, 2001 
demanderait un effort considérable et une solide confiance dans les pouvoirs de l'interprétation. Au sein d'un article choisi, il est sans doute un peu moins difficile de dégager le sens des sélections (de tels mots et phrases plutôt que de tels autres). Deux exemples. Quand une citation perturbe la syntaxe de la phrase-source, il faut sans doute y voir un geste fort : le « We will show the world that we will pass this test. God bless », de Bush, est transformé en «We will show the world / God bless ». Il en va de même quand Vinaver accentue le pathétique d'une description du journaliste, passant de «People [...] stepping over rubble, made the full trip to safety » à «People [...] / Stepping on bodies and rubble / Made the full trip to safety ». Mais affirmer que les effets visés sont la ridiculisation de Bush et la critique d'un discours journalistique voyeuriste, serait franchir une étape herméneutique de façon indue. Il faudrait pour cela un réseau de faits de ce genre; pris singulièrement, ce ne sont que des « indices ${ }^{11}$.

Lorsqu'à partir d'un article long, Vinaver ne prélève qu'une courte citation, on pourrait distinguer un sous-ensemble, en parlant de «tronquage» (mot de Vinaver, qui distingue ce terme de celui d'"émincissage», quand les suppressions sont moins importantes et plus harmonieusement réparties ${ }^{12}$ ). Par exemple, Michel Vinaver est parti d'un article du Washington Post de 2800 mots $^{13}$, dont il avait commencé par faire cette version :

\author{
$<$ JOURNALIST $>$ \\ The 19 hijackers <men who hijacked the four planes < airliners $>$ simultaneously> \\ Who carried out the worst act of terrorism in history $<$ worked $>$ \\ Worked with little outside help \\ As a single, integrated group \\ Composed of identifiable leaders \\ And shadowy foot soldiers \\ Who prepared for their final day \\ In a tight choreography \\ Over 18 months. \\ Only one aboard each of the four commandeered aircraft \\ Knew how to fly a plane \\ Three of the four suspected pilots had roomed together \\ In Germany, where they \\ Attended the Technical University of Hamburg \\ Their preparations were imperfect \\ Some $<$ in that group $>$ were kicked out of pilot schools \\ Some had to pay cash for their plane tickets after their credit cards were rejected \\ Two were late for the Boston flight that would be the first to slam into the World Trade Center \\ But inexact as it was \\ Their plot succeeded in killing <implementing $>$ \\ More than 6,000 people $<$ The worst act of terrorism in history ${ }^{14}$
}

\footnotetext{
11 On pense toutefois à une autre citation, dans Portrait d'une femmme de Vinaver (1984) : l'avocat de la partie civile cite Malraux (Les conquérants), pour qui «juger c'est de toute évidence ne pas comprendre puisque, si l'on comprenait, on ne pourrait pas juger », mais tronque la phrase après "comprendre ». Marianne Noujaïm, auteur de la première thèse consacrée à l'œuvre de Michel Vinaver, montre bien comment Vinaver déprécie le discours de l'avocat, qui dénature complètement la sentence originale : « la maxime se présente comme un constat absolument dépourvu de la dimension normative (inverse) que lui confère Lubet dans son discours " (Du dialogisme à l'esthétique polyphonique dans le théatre de Michel Vinaver. Approche rhétorique et poétique, déc. 2009, p. 137).

12 Pour une présentation de ce vocabulaire de la réécriture, voir Simon Chemama, « Les genèses de Pardessus bord», Genèses théâtrales, ITEM-CNRS, notamment p. 164-170.

13 Amy Goldstein, «Hijackers Led Core Group », Washington Post, 30 septembre 2001, page A01 (en ligne : http://www.washingtonpost.com/ac2/wp-dyn?pagename=article\&contentId=A468922001Sep29\&notFound=true)

14 Brouillon de 11 septembre 2001, manuscrit (IMEC - VNV 35.5).
} 
Il compose ensuite la version finale, en deux répliques du « journaliste » de trente mots au total :

\author{
JOURNALIST \\ The nineteen men prepared for their final day \\ In a tight choreography \\ Over eighteen months \\ $[\ldots]$ \\ JOURNALIST \\ Only one aboard each of the four commandeered aircraft \\ Knew how to fly a plane ${ }^{15}$
}

Que conserve-t-il ? Dans la première esquisse, Vinaver avait été sensible aux menus incidents qui émaillèrent le parcours des terroristes (renvoyés de l'école de pilotage, en retard pour le vol...). Puis il semble se concentrer seulement sur les chiffres (19 et 18, 1 sur 4), ainsi que sur la résonnance artistique du mot « choregraphy »- la réplique devient plus abstraite.

La deuxième technique pourrait être celle du collage, dans son sens pleinement esthétique (juxtaposition - valant pour elle-même - d'éléments hétérogènes ${ }^{16}$ ). C'est particulièrement sensible quand ce collage se réalise à l'intérieur d'une même réplique. Les chorus en constituent les exemples les plus saisissants. En les examinant, on comprend que la forme même du journal détermine en partie celle de la pièce. Considéré avant tout par l'auteur dans sa matérialité, et non comme support signifiant logique, l'objet journal (de par sa mise en page) détermine l'agencement textuel de la pièce ${ }^{17}$. Dès lors, certaines sources de 11 septembre 2001 sont impossibles à identifier si l'on se sert seulement des archives Internet des journaux. Il faut consulter les archives papier, car certaines répliques viennent des encarts publicitaires, ou des légendes sous les photos. Dans le cas de la première intervention du chorus, Vinaver compose une réplique à partir de différents éléments que l'on trouvait sur deux pages de l'International Herald Tribune du 10 septembre 2001 :

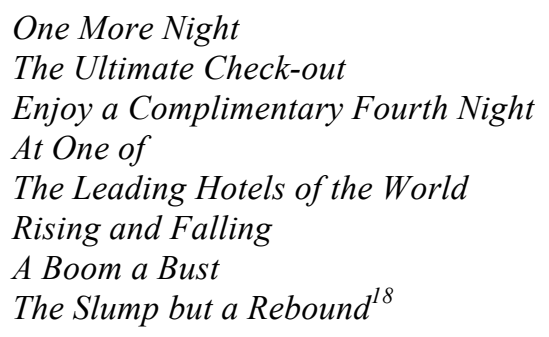

Les quatre premiers vers correspondent, comme on l'entend assez clairement, à une publicité, sans rapport sémantique ni logique avec les autres phrases. C'est un encart que l'on trouvait sur la page 2 du quotidien, pour The Leading Hotels of The World (« One More Night. The Ultimate late check-out. Enjoy a complementary fourth night with "Leading offers" »). Mais les trois vers suivants ont une provenance différente : ils sont issus de titres d'articles du même numéro,

1511 septembre 2001, p. 64. Les deux répliques du journaliste sont séparées par quatre autres répliques (d'Atta et du premier trader).

16 On peut rappeler la distinction que faisait Anne Ubersfeld (Lire le théâtre, Paris, Éditions sociales, 1977, note 48) entre collage et montage : «Il y a montage quand les éléments hétérogènes prennent sens par la combinaison qui est faite avec eux; il y a collage quand c'est l'hétérogénéité qui fait sens, non la combinaison. »

${ }^{17}$ Voir le livre que Marie-Ève Thérenty consacre à l'influence réciproque de la littérature et du journal au $\mathrm{XIX}^{\mathrm{e}}$ siècle (La littérature au quotidien. Poétiques journalistiques au XIX siècle, Seuil, 2007). Les critères d'actualité, d'effet-rubrique et de collectivité propres à la "matrice médiatique » seraient tout à fait pertinents pour analyser l'œuvre de Vinaver.

${ }^{18} 11$ September 2001, op. cit., p. 136. 
et de la rubrique editorial / opinion (articles qui, forcément, n'ont rien à voir avec les attentats du 11 septembre). "Rising and Falling » était le titre exact de l'édito ; "A Boom a Bust» est tiré d'un article intitulé « Where There Was a Boom There Is Now Going to Be a Bust » de James Grant $^{19}$; enfin, « The Slump but a Rebound» est également le condensé d'un titre d'article, de David Ignatius, «The Slump Is Here, but a Rebound Is in the Cards » ${ }^{20}$.
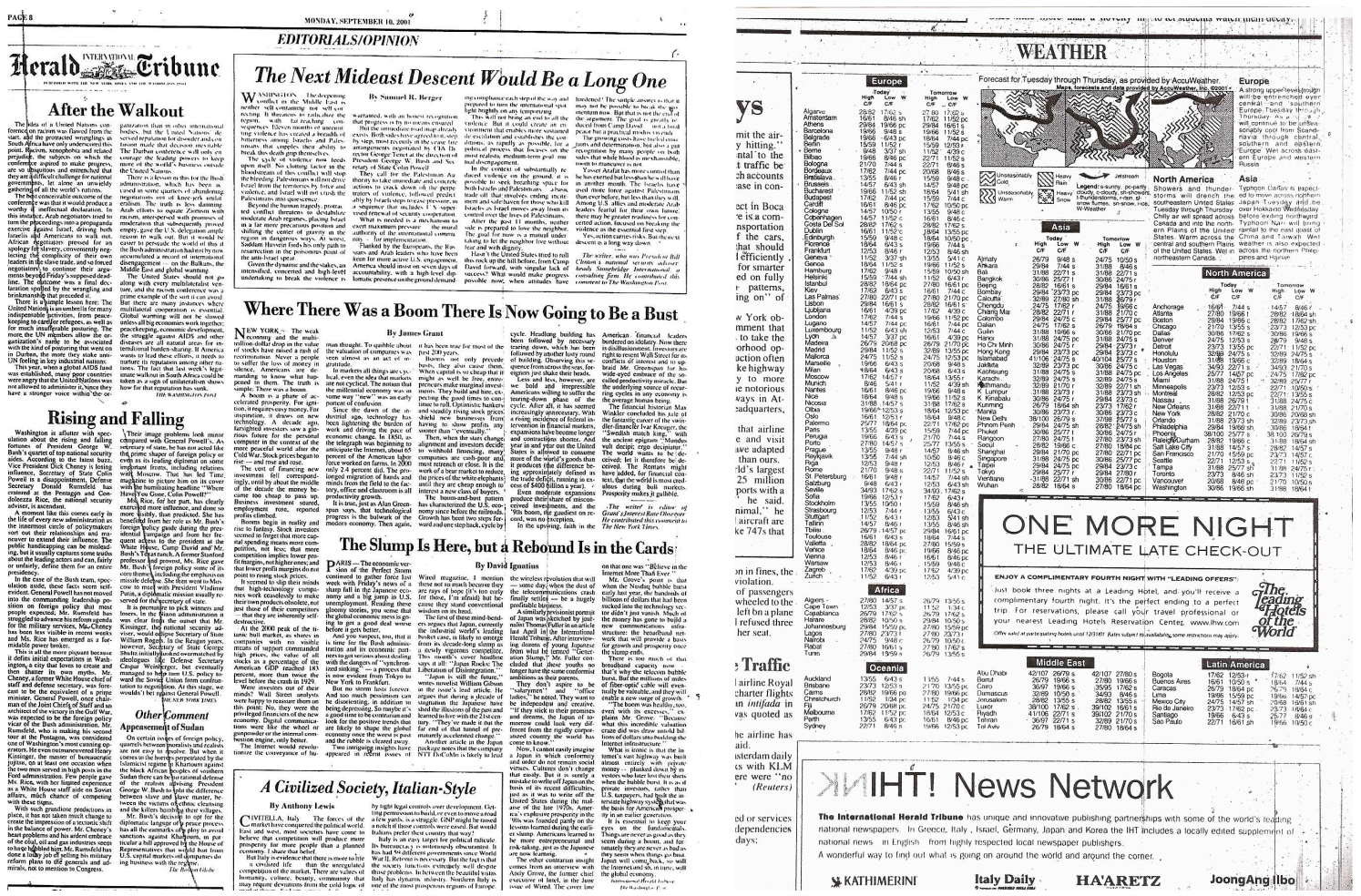

IHT, 10 septembre 2001, p. 8 (extraits)

IHT, 10 septembre 2001, p. 2 (extraits)

Accessoirement, Vinaver a-t-il voulu, en se servant de ces textes antérieurs aux attentats, faire référence, dans ce chorus, au chant choral antique, parfois porteur des présages (mais de présages que personne n'entend) ? Les 9 et 10 septembre 2001, les titres annonçaient une catastrophe - on pourrait les traduire de cette façon : "Ce qui s'élève puis qui s'affaisse », « Une explosion un effondrement», "La chute mais un rebond». Et l'avertissement des titres (et même de la publicité : "One more night» - il ne reste plus qu'une nuit...) contraste avec l'aveuglement des journalistes (il ne peut en être autrement); David Ignatius constate la dégradation des économies japonaise et américaine, mais affirme: "Maybe it's a good time to be contrarian and look for the positive trends that are likely to shape the global economy once the worst is past and the rubble is cleared away ${ }^{21}$.

On voit bien, déjà, la limite du classement qu'on propose (ou plutôt de l'idée d'« intervention » de l'auteur) : la décontextualisation très forte dont font l'objet ces phrases, placées dans un nouveau cadre, correspond en fait à une intervention considérable (maximale, dirions-nous - alors même qu'il n'y a que peu ou pas du tout de transformations sur les

19 Article paru sous le titre : «Sometimes the Economy Needs a Setback» dans le New York Times du 9 septembre 2001. Il commente le phénomène de chute des valeurs boursières consécutive aux phases de hausses spéculatives (bulles). Le Dow Jones avait progressé considérablement jusqu'en 1999, avant de perdre près d'un tiers de sa valeur.

http://www.nytimes.com/2001/09/09/opinion/sometimes-the-economy-needs-a-setback.html

20 Article paru sous le titre : « No Slump Lasts Forever » dans le Washington Post du 9 septembre 2001.

21 Ibid., c'est nous qui soulignons (« rubble », les débris, est un mot très important dans le lexique du 11 septembre). 
morceaux prélevés). Le collage réalisé par Vinaver s'apparente à un geste artistique fort, semblable, dirions-nous, à celui de Schwitters : l'artiste allemand prélevait des fragments du monde réel (ticket de métro, bout de chiffon...) puis les « redistribuait ${ }^{22}$ sur une toile (aux dimensions, en général, aussi modestes que celles de la pièce 11 septembre 2001). Dans un premier état de son travail, Vinaver avait prévu de regrouper ces phrases au début (sous forme d'une longue liste, ou poème), en en faisant un «Prélude». Nous recopions ici la deuxième page $^{23}$ :

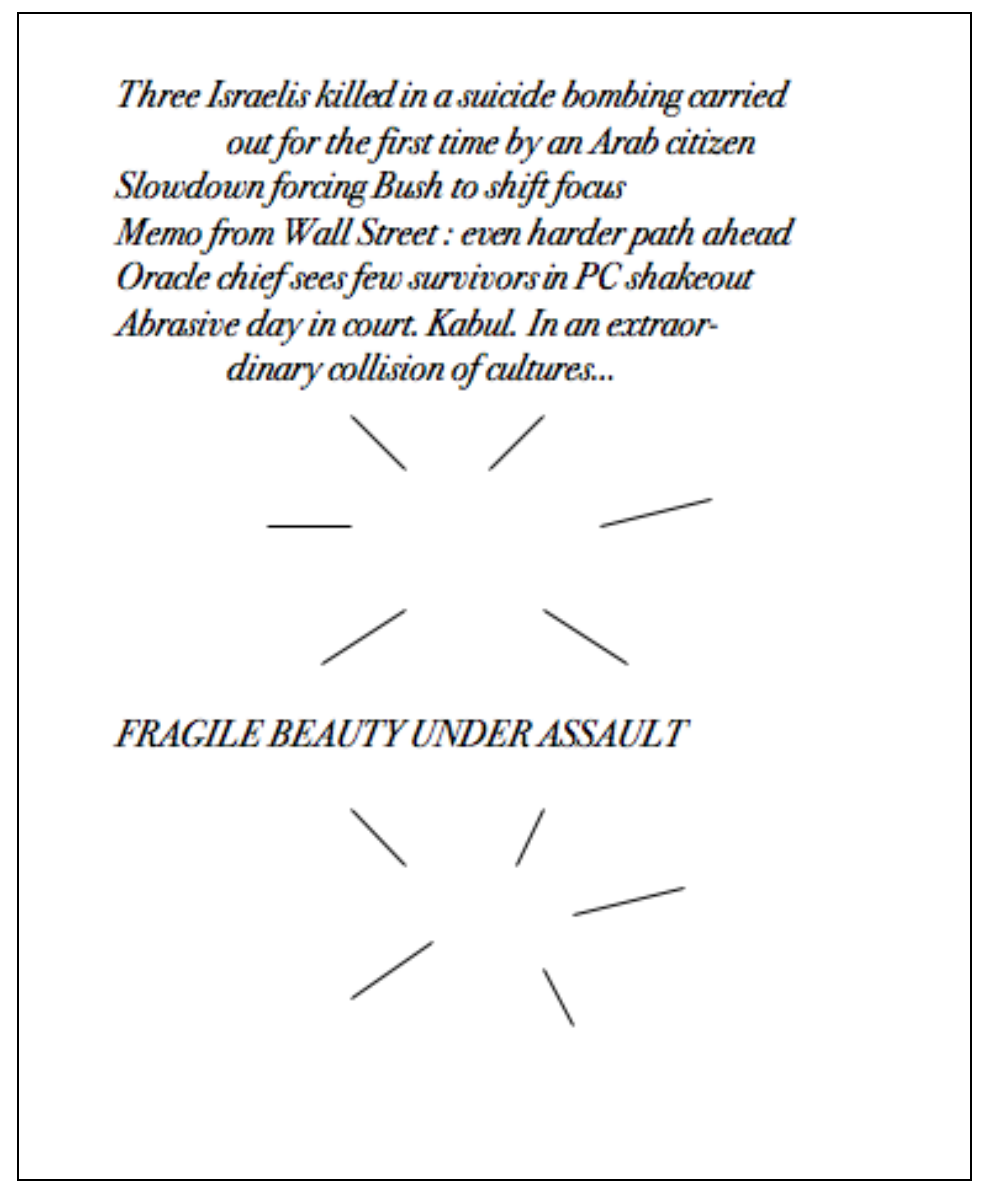

L'effet aurait-il été différent? En choisissant de les disséminer tout au long de la pièce Vinaver les «redistribue », et évite peut-être de construire deux parties trop distinctes: la deuxième aurait été bien plus réaliste que la première. Le terme de "prélude », dans le lexique de la musique, a deux sens que Vinaver semble combiner. Le premier (historiquement) n'est pas «pièce instrumentale ou orchestrale de forme libre servant d'ouverture à une œuvre vocale (opéra, oratorio) ou instrumentale (suite) », mais "suite de notes, souvent improvisée, chantée ou jouée pour se mettre dans le ton et essayer, chauffer la voix ou l'instrument $»^{24}$. Dans la

22 Voir Jean-Christophe Bailly, Kurt Schwitters, Paris, Hazan, 1993, p. 6. «C'est par la distribution qu'advient la métamorphose où chaque élément, quoique relié à son origine, en même temps s'en délie, venant se déposer dans un nouveau mode de relations » (Ibid., p. 77).

23 Quatre pages manuscrites (IMEC - VNV 35.2). Les petits traits sont la figuration des explosions lors des crashs des avions.

24 http://www.cnrtl.fr/definition/prélude. Dans À la renverse (pièce de 1979, où l'on trouvait déjà un «Prélude» suivi d'une «Fugue » près de trois fois plus longue), la première indication scénique du Prélude disait : «Les comédiens accèdent sur le plateau dans leur vêtements de tous les jours, ils se mettent en train : exercice du geste et de la parole, chacun pour soi. Leur propos s'insèrent comme des éclats dans le corps du récit. Source: Merce Cunningham, musiciens d'orchestre accordant leurs 
version éditée de 11 septembre 2001, ces «éléments choraux 》 (comme il les désigne) semblent jouer un rôle dramaturgique, comme vecteurs de transition d'une séquence à l'autre de la pièce ${ }^{25}$.

Continuons tout de même notre imparfait classement, et repérons, au sein de ce deuxième ensemble, une variante qui serait la recomposition, qui consiste aussi en un prélèvement, mais avec un placement des phrases dans un ordre nouveau. Il en va ainsi dans la longue réplique de Todd Beaver du début. Michel Vinaver écrit par exemple: «Yes this includes the two pilots who are injured / They've stabbed one passenger to death ", alors que, dans l'article, l'information est donnée dans l'ordre inverse ${ }^{26}$.

La troisième technique est l'ajout. Toujours dans ce dialogue entre Beaver et Lisa, Vinaver ajoute plusieurs détails, comme celui-ci :

For God's sake this is incredible you've got the same name as my wife

Listen Lisa my name's Beaver Todd Beaver

C'est lui qui mentionne cette identité (qui inscrit dans la pièce elle-même cette notion théorique de sameness of spelling...) entre le nom de la femme de Todd et celui de sa dernière interlocutrice. On reconnaît là l'attirance profonde de Vinaver pour ce genre de coïncidence ${ }^{27}$. À notre connaissance, Michel Vinaver ne disposait pas de la retranscription complète de cette conversation. Il reconstitue donc un dialogue (ajoutant des répliques brèves comme : "Oh you have ? »), qui forme l'un des passages les plus dramatisés de la pièce. L'introduction du journaliste ne se trouvait pas non plus dans l'article-source :

Here I am standing

At Ground Zero I behold

Scenes of chaos and fear

Some are alive some are dead

some were alive

Tout comme cette remarque :

Mohamed Atta when he wrote his

will five years

earlier

Did not know

That there would be no body

Pour un plus large aperçu du travail de copie et réécriture, donnons l'exemple du passage le plus complexe de la pièce, qui met en jeu la plupart de ces techniques. Il s'agit de la

instruments. » (À la renverse, Lausanne, Éditions de l'Aire, 1980, p. 13. Cette indication disparait dans la nouvelle version de la pièce).

${ }^{25}$ Sauf dans un cas où le chorus dialogue avec Bush (11 septembre 2001, p. 144-148).

26 Charles Lane, Don Phillips et David Snyder, «A Sky Filled With Chaos, Uncertainty and True Heroism; Passenger on One Plane Relayed Plan; Controllers Scrambled to Track Flights», The Washington Post, 17 septembre 2001.

${ }^{27}$ Ironie ou harmonie ? Dans le fait divers qui est à l'origine de L'Ordinaire, c'est au-dessus du « col du Planchon» que l'avion rencontre une zone de perturbations. Michel Vinaver n'a pu qu'être frappé par le nom de ce col, à la frontière de l'Argentine et du Chili, homonyme du metteur en scène lyonnais qui avait créé Les Coréens et Par-dessus bord. Là encore, on pourrait faire le rapprochement avec Schwitters, qui " prélève ce qui pour lui est ressemblant, ce qui fait sens en vue d'un sens qu'il est seul à pressentir. Ainsi le sens se constitue-t-il en amont de l'œuvre comme un réseau d'indices dispersés, ayant chacun une présence autonome, mais se trouvant avec tous les autres dans un rapport de connexions que le "collectionneur" seul est capable de déceler » (J.-C. Bailly, Schwitters, op. cit., p. 72). 
séquence d'interview au sujet de l'ascenseur bloqué. Nous recopions ci-dessous l'article du New York Times, avec la représentation des suppressions, reformulations ou ajouts effectués par Michel Vinaver ${ }^{28}$ :

\section{$<$ JOURNALIST $>$}

Now memories orbit around small things. / None of the other window washers liked his old green bucket, / but Jan Demczur, whowerked inside 1 World Trade Center, / found its rectangular mouth / perfect for dipping and wetting his squeegee / in one motion. So on the morning of the 11th, as he waited at the 44th floor Sky Lobby to connect with elevators for higher floors, bucket and squeegee dangled from the end of his arm.

The time was 8:47 a.m. With five other men - Shivam Iyer $\rightarrow$ Shivan Iyen, John Paczkowski, George Phoenix, Colin Richardson / and another man whose identity could not be learned -- / Mr. Demczur (pronouneed DEM-sir) boarded Car 69-A / an express elevator that stopped on floors 67 through 74 .

The car rose / But before it reached $<$ the level of $>$ its first $<$ potential $>$ landing

\section{$<$ DEMCZUR $>$}

We felt a muted thud," Mr. Iyer said. " / The building shook / The elevator swung from side to side / like a pendulum

\section{$<$ IYER>}

Then it plunged / In the car, someone punched an emergency stop button. / $<$ The elevator cabin stopped $>$ At that moment $8: 48 \mathrm{a} . \mathrm{m} .1$ World Trade Center had entered the final 100 minutes of its existence. No one knew the clock was rinning, least of all the men trapped inside Car 69-A; they were as cut off 500 feet in the sky as if they had been trapped 500 feet underwater.

They did not know their lives would depend on a simple tool.

After 10 minutes, a live voice delivered a blunt message over the intercom. There had been an explosion. Then the intereom went silent. / Smoke seeped into the elevator cabin. One man cursed skyserapers. Mr. Phoenix, the tallest, a Port Authority engineer, poked for a ceiling hateh.

$<$ JOURNALIST $>$

Others pried $\rightarrow$ They succeeded in prying apart the car doors,propping them open with the long wooden handle of Mr. Demezur's sque gee.

$<$ IYER $>$

But There was no exit.

They faced $\rightarrow$ Facing us a $<$ blank $>$ wall, stenciled with the number "50." That particular elevator bank did not serve the 50 th floor, so there was no need for an opening. To eseape, they would have to make one themselves.

\section{$<$ JOURNALIST $>$}

Mr. Demczur felt the wall. Sheetroek. Having worked in constrution in his early days as a Polish immigrant, he knew that it could be cut with a sharp knife.

No one had a knife.

From his bucket, Mr. Demezur drew his squeegee. He slid its $\rightarrow$ Sliding his squeegee's metal edge against the wall, back and forth, over and over. He was spelled by the other men. Against the smoke, they breathed through handkerchiefs dampened in a container of milk Mr. Phoenix had just bought.

Sheetrock comes in panels about one inch thick, Mr. Demczur recalled. They cut an inch, then two inches. Mr. Demezur's hand ached. As he carved into the third panel, his hand shook, he fumbled the squeegee and it dropped down the shaft.

He had one tool left: a short metal squeegee handle. They carried on, with fists, feet and handle, $<$ was able to $>$ cutting an irregular rectangle about 12 by 18 inches $\rightarrow 30$ by 45 centimeters. Finally, they hit a layer of white tiles. A bathroom. They broke the tiles.

One by one, the men squirmed through the opening, headfirst, sideways, popping onto the floor near a sink. Mr. Demezur turned back. "I said, 'Pass my bucket out,' " he recalled.

$<$ IYER>

By then, about 9:30 $\rightarrow$ It was nine thirty by this time, the 50th floor was already deserted; except for firefighters, astonished to see the six men emerge. "I think it was Engine Company 5,"

${ }^{28}$ Article de Jim Dwyer, «Fighting for Life 50 Floors Up, With One Tool and Ingenuity », New York Times, 9 octobre 2001. Toutes les marques de ponctuation sont supprimées (sauf les points d'interrogation). Vinaver ajoute aussi de nombreux passages à la ligne (notés : / ). Les mots et phrases rayés sont ceux que Vinaver n'a pas retenus ou a reformulés, ceux en gras (précédés d'une flèche $[\rightarrow]$ ) ont subi une reformulation, et ceux entre crochets ont été ajoutés. 
Mr. Iyer said. "They hustled us to the stairease."

On the excruciating single file descent through the smoke, someone teased Mr. Demczur about bringing $\rightarrow$ wouldn't drop his bucket $<$ as we stumble down in the rubble>. "The company might not order me another one," he replied $\rightarrow$ said.

$<$ JOURNALIST $>$

At the 15th floor, Mr. Iyer said: "We heard a thunderous, metallic roar. I thought our lives had surely ended then." The south tower was collapsing. It was 9:59. Mr Demezur dropped his bucket. The firefighters shouted to hurry. $<$ As the descent was getting rougher requiring the use of hands roundabout the twenty-first floor he had to let it go $>$

At 23 minutes past $10 \rightarrow$ ten twenty-three, they burst onto the street, ran for phones, sipped exygen and, five minutes later, fled as the north tower collapsed. / Their escape had taken 95 of the 100 minutes <between the plane's crash and the buildng's collapse>. "It took up to one and a half minutes to clear each floor, longer at the lower levels," said Mr. Iyer, an engineer with the Port Authority.

$<$ IYER $>$

"If the elevator had stopped at the $60^{\text {th }}$ floor, instead of the $50^{\text {th }}$ floor, we would have been five minutes too late.

"And that man with the squeegee. He was like our guardian angel."

Since that day, Mr. Demezur has stayed home with his wife and children. He has pieced together the faces of the missing with the men and women he knew in the stations of his old life: the security guard at the Japanese bank on the 93rd floor, who used to let him in at 6:30; the people at Carr Futures on 92; the head of the Port Authority. Their faces keep him awake at night, he says.

His hands, the one that held the squeegee and the other that carried the bucket, shake with absence

Ce type de présentation est sans doute peu digeste, mais il nous permet de voir au plus près comment s'élabore le texte. Alors même que dans cet extrait la part du texte "inventé » est proportionnellement plus importante que dans le reste de la pièce, on comprend que Vinaver se réapproprie une matière discursive en la littérarisant. Deux remarques évidentes : Vinaver remplace les nombres écrits en chiffres par des mots en toutes lettres ; mais surtout il passe à la ligne fréquemment (ce que nous n'avions pas encore précisé), donnant au texte une dimension poétique certaine - faut-il parler de vers libres? de versets? d'unités rythmiques $?^{29}$ D'autre part, il adapte cette matière au genre théâtral, mais aussi à la scène : il crée un texte à plusieurs énonciateurs ${ }^{30}$ qui prend la forme d'un dialogue théâtral, même si les voix ne s'adressent pas directement les unes aux autres; il raccourcit considérablement le texte-source, et il le rend plus clair, en supprimant certains détails techniques, comme le déroulé précis du travail de Demczur. On peut aussi avoir l'impression que Vinaver cherche à créer une certaine unité, ou du moins des échos, entre les différentes séquences de sa pièce (tirées, rappelons-le, d'environ trente articles ou discours différents) : en choisissant le mot « rubble» dans la reformulation d'une phrase ( « as we stumble down in the rubble »), Vinaver utilise un mot employé à trois autres reprises dans la pièce ${ }^{31}$. Enfin, la seule phrase entièrement « inventée » (composée par Vinaver lui-même) concerne Demczur, et c'est sur la comparaison de Demczur à un « ange gardien » que se termine l'adaptation, c'est-à-dire sur le climax des témoignages, alors que la fin de l'article-source introduisait une autre temporalité, celle de l'interview réelle faite plusieurs jours après l'événement.

29 Barbara Métais-Chastanier a tout à fait raison de comparer cette mise en forme à celle opérée par Reznikoff ainsi que par Weiss ( $c f$. «Témoigner pour le réel », en ligne dans ce même hors-série, http://agon.ens-lyon.fr/index.php?id=1787).

30 Ici il n'a pas eu à transformer les paroles des rescapés, puisque le journaliste les rapportait au discours direct. Globalement, il faut remarquer une chose : les articles utilisés sont déjà des articles qui agglomèrent les paroles, des articles citants. Ce qui n'est sans doute pas à négliger dans la dynamique générale de l'écriture de la pièce.

3111 Septembre 2001, p. 154, 158 et 166 (chorus déjà cité). 
La dernière technique est l'invention d'un passage. La dernière réplique (celle de la jeune femme qui n'est pas allée travailler parce que son fils était malade) est la seule à ne pas avoir été extraite d'un article ou d'un discours existant, mais simplement inventée. Il est amusant de voir qu'en termes de volume, le rapport entre le cité et l'inventé est inverse à celui d'un texte littéraire «normal ». Mais nous avons aussi l'intuition que le sens de la pièce aurait été différent si cette réplique n'avait pas été écrite. Était-elle nécessaire (comme la moindre tige sur laquelle toutes les citations peuvent se greffer - plante monstrueuse) ? Constitue-t-elle le discours de la pièce, face auquel les discours prélevés viennent résonner? En tout cas, elle contribue à donner de 11 septembre 2001 l'image d'un texte complètement ouvert, semblable au monde lui-même, dans lequel on trouverait, entre autres, une citation de Vinaver.

Quel serait le terme idéal pour caractériser ces reprises ? Le mot « adaptation » (dont nous venons de nous servir) convient-il ? Il est vrai que le rapport au texte-source nous fait penser à celui que Vinaver a entretenu avec les deux pièces qu'il a adaptées : La Fête du cordonnier (1959) ${ }^{32}$ et Les Troyennes (2002), à la différence de celles qu'il a «traduites »: Le Suicidé, Les Estivants, Jules César, et Le Temps et la Chambre. Vinaver a déjà expliqué comment il réalise ses adaptations ${ }^{33}:$ il traduit le texte une première fois (même à l'aide de traductions existantes quand il ne connaît pas bien ou pas du tout la langue, comme c'est le cas $\mathrm{du}$ grec ancien ${ }^{34}$ ), puis il attend un moment (laisse reposer) et reprend ensuite cette traduction en la retravaillant comme s'il s'agissait du premier jet d'une de ses propres pièces. Or, ces deux adaptations, Vinaver finit par les concevoir comme ses propres textes: "Pour Les Huissiers, j'ai décalqué [1'histoire] d'Edipe à Colone. La Fête du cordonnier est tirée d'une pièce de Dekker... ${ }^{35}$. Dire de cette pièce qu'elle est seulement « tirée » de la pièce de Dekker est étonnant (et aucun éditeur ne se risquerait à présenter les choses de cette façon sur la couverture de l'ouvrage) ${ }^{36}$. Mais cela pose en fait des questions déterminantes sur ce qu'est la littérature. Qu'est-ce qui détermine la paternité d'un texte? De quelle façon peut-on être ou rester le propriétaire d'un texte, et à quel moment y a-t-il passage d'un auteur à un autre ? Comment distinguer plagiat et hypertextualité ?

\section{La question du plagiat se pose-t-elle?}

D’un point de vue juridique, ces questions ont déjà été examinées. Les lois du droit d'auteur s'appliquent aussi au journalisme. Or, Vinaver ne cite pas précisément ses sources et ne met jamais de guillemets. Il ne faut pas oublier, certes, que depuis 1969 (Par-dessus bord),

\footnotetext{
32 Vilar demande à Vinaver d'adapter cette pièce, alors que deux traductions venaient de paraître : celle d'A. Koszul pour Les Belles-Lettres (1955, en alexandrins), et celle de J. Loiseau chez Aubier (1957).

${ }^{33}$ Voir notamment « De l'adaptation » (1959), repris dans Écrits sur le théâtre 1, Paris, L’Arche, 1982, p. 82 : «Dekker est là tout entier, et je suis, moi, là aussi, tout entier. Comment s'explique, et comment se justifie, pareil larcin?»

${ }^{34}$ Voir « Le théâtre comme objet fractal », entretien avec J.-L. Rivière, in Europe n ${ }^{\circ} 924$, avril 2006, p. 127 sq. Ces questions d'adaptation et de traduction sont également développées dans l'article de Sylvain Diaz et Anne-Sophie Noel paru dans le dossier (http://agon.ens-lyon.fr/index.php?id=1782).

${ }^{35}$ Michel Vinaver, «Auto-interrogatoire » (1973), in Écrits ... 1, op. cit., p. 308.

${ }^{36}$ On dit généralement qu'une œuvre est, par exemple, tirée d'un fait divers - cela signifie qu'elle part de là, s'en inspire. Quand on affirme qu'une œuvre est tirée d'une autre œuvre, c'est qu'il y a au moins ce que Genette appelle une dérivation extramodale: comme lorsqu'une pièce est tirée d'un roman (alors que dans le cas de La fête du cordonnier, il y a pleine identité modale).
} 
Vinaver a supprimé de ses pièces toute ponctuation, guillemets compris. C'est une façon de ne pas distinguer de niveaux d'énonciation ${ }^{37}$ - et de renforcer l'impression si forte dans son théâtre que tout est sur un même plan d'immanence. Toutefois, il ne cherche pas non plus à dissimuler quoi que ce soit. Dans les exemples que donne Hélène Maurel-Indart, spécialiste du plagiat en littérature, on ne trouve pas de pures copies comme celles de 11 septembre 2001, mais toujours des reprises déguisées (noms changés, adjectifs supprimés, ordre inversé, etc...) - et ce sont avant tout les idées, les actions ou les situations qui sont reprises et qui font ensuite l'objet d'attaques judiciaires.

Un des arguments de la défense dans les affaires de plagiat est souvent celui de la «banalité» d'une phrase ou d'une situation - argument spécieux, qui réintroduirait des distinctions de contenu aristotéliciennes, dont la littérature est sortie depuis le XIX ${ }^{\mathrm{e}}$ siècle (de plus, le critère de «banalité » mettrait de fait le théâtre du quotidien à l'abri de toute condamnation, ce qui serait illogique). Le critère de la «quantité » des emprunts est bien plus objectif, et l'on peut très bien imaginer un système complexe identifiant une limite à ne pas dépasser (en pourcentage du volume global de l'œuvre copiée et de l'œuvre produite) - mais celui-ci n'a jamais été mis en place. Enfin, Hélène Maurel-Indart montre bien (à partir de procès réels) que la qualification de plagiat dépend de la nature du texte-source. Si «l'emprunt d'éléments documentaires bruts » est tout à fait toléré, « l'emprunt de matériaux faisant l'objet d'une réflexion personnelle et issus d'un travail de décryptage, d'analyse et de réappropriation créative » ne l'est pas ${ }^{38}$. Dans 11 septembre 2001, pour schématiser, il y aurait peut-être deux cas de figure à distinguer. La parole des journalistes, d'une part, qui, sans être très riche en «analyses », reste une œuvre de l'esprit, sur laquelle s'appliquent les lois de la propriété intellectuelle. Et d'autre part, la parole des chefs politiques (Bush, Ben Laden) et celle des témoins : les discours qu'ils ont produits ne leur font pas gagner d'argent. S'ils devaient se plaindre, ce ne serait donc pas pour des questions de droits d'auteur, mais éventuellement de détournement de leur propos, de diffamation, etc. Extraire d'un discours des bouts de phrase, dans de nombreux contextes (un article de journal par exemple), c'est en effet une erreur (qui peut devenir une faute) - la question est de savoir ce qui change lorsque cette soustraction est le fait d'un écrivain. Quand Rumsfeld, qui n'a qu'une réplique dans la pièce, affirme : « Ultimately they're going to collapse from within / That is what will constitute victory », on sait peut-être qu'il parle des terroristes et de leur organisation, mais dans la juxtaposition immédiate avec les autres répliques, on a l'impression qu'il parlait des tours ${ }^{39}$ ! C'est le pouvoir de l'ironie.

${ }^{37}$ Niveaux d'énonciation que la bathmologie de Barthes se proposait d'étudier (Roland Barthes, Seuil, 1975). Des guillemets, on en trouve dans les trois premières pièces de Vinaver. Mais ils encadrent toujours les citations d'éléments discursifs intra-diégétiques (fictifs - dans Iphigénie Hôtel, par exemple, les personnages citent le regretté M. Oreste ou bien lisent à haute voix un article de journal, respectivement p. 114 et 167), et non de discours réels ou tirés d'œuvres littéraires. L'intertextualité fonctionne différemment : de façon générale, peut-elle s'accommoder de ces marques de renvoi ? Nous n'avons pas d'exemples en tête. Reste le cas où c'est le personnage lui-même qui, dans une posture d'acteur ou de sophiste, cite un grand auteur. On a déjà évoqué le cas de Lubet-Malraux ; on trouve dans la même pièce ce vers de Racine cité par Sophie: «Je me livre en aveugle au destin qui m'entraîne » (Portrait d'une femme, op. cit., p. 36, sans guillemet). Dans Par-dessus bord, c'est Jack, qui cite dans une même réplique saint Augustin, Nietzsche et Swift. La première est le fait d'une « comédienne » (comme le dit sa concierge), la seconde d'un bonimenteur inspiré.

38 Hélène Maurel-Indart, Du plagiat (1999), nouvelle édition en Folio Essais, 2011, p. 98. Elle fait cette distinction à partir de deux procès qui ont eu lieu en 2001 : l'un contre Chimo (Lila a dit ça), qui a été acquitté ; l'autre contre Alain Minc (pour Spinoza, le masque de la sagesse), qui a été condamné.

3911 septembre 2001, p. 153. « À la fin ils s'effondreront de l'intérieur / c'est cela qui constituera la victoire » (traduction de Michel Vinaver). Citation tirée de la conférence de presse de Rumsfeld et du 
De toutes façons, dans ces procès pour plagiat, les jugements se font avant tout sur le « sentiment » du juge à la lecture de l'œuvre ; un même texte, en deux lieux différents, ou à deux dates différentes, ne sera pas jugé de la même façon. Aussi serait-il vain de chicaner. Ce qui demeure, toutefois, c'est la mauvaise presse du copier-coller aujourd'hui - alors que l'imitation de modèles fut la norme de l'écriture humaniste et classique et le collage celle de la révolution moderniste. Marie Darrieussecq, violemment accusée par deux autres romancières, a publié un livre sur ces questions de plagiat et d'imitation ${ }^{40}$. "Faut-il, à la fin des romans, écrire une bibliographie ? » se demande-t-elle ${ }^{41}$. Vinaver en a donné une, partielle, à la fin d'une seule de ses pièces: pour Iphigénie Hôtel (1959). Il donnait sa «source» principale (Amour de Henry Green), et ajoutait: "j'ai puisé aussi, directement ou indirectement, notamment chez Raymond Guérin (L'Apprenti), T. S. Eliot (Sweeney Agonists), Rossellini (Voyage en Italie) » et chez Tchekov ${ }^{42}$. Il y avait comme un geste poétique dans cette petite énumération aux références précises, mais aux termes plutôt vagues ("puisé », « directement ou indirectement»), qui devenait presque un élément à part entière du texte littéraire. Quant aux reprises en question, il s'agissait avant tout de situations ou éléments d'intrigue, de thèmes ou d'idées, plutôt que de répliques précises. Dans 11 Septembre 2001, ce sont les mots eux-mêmes, la matière même de la littérature, qui sont recopiés.

On sent une certaine résistance de l'auteur à avouer pleinement l'origine du texte anglais, résistance qui n'est peut-être pas entièrement étrangère à ces problématiques socioculturelles (l'imitation interdite, ou l'imitation vue comme limitation). Le théâtre verbatim est aujourd'hui la grande tendance (principalement anglo-saxonne) d'un théâtre du réel. Comme son nom l'indique, il s'agit de pièces composées mot pour mot d'extraits de documents réels (rapports d'audiences, discours politiques, entretiens, journaux intimes...). Certains critiques tentent d'inclure la pièce de Vinaver dans ce courant. Mais si 11 septembre 2001 peut se rapprocher formellement des pièces verbatim (des meilleures d'entre elles - celles qui ne font pas l'économie d'un travail poétique), celles-ci ont toujours pour principe de mentionner précisément les sources utilisées, ce qui nous semble constituer une différence majeure ${ }^{43}$.

Vinaver a-t-il même voulu, in extremis, marquer davantage la pièce de son empreinte ? Comme on l'a déjà remarqué, la dernière réplique est une exception, en tant que seule réplique inventée. Or, on s'aperçoit qu'elle ne figure dans aucun des brouillons de la

général Myers à propos de l'opération «Enduring Freedom». Rumsfeld parlait bien des terroristes (même si l'antécédent du pronom «they » était «terrorism » et qu'il y avait donc une petite rupture syntaxique). $\quad C f . \quad$ http://www.defense.gov/transcripts/transcript.aspx?transcriptid=2011. L'intention n'est pas la même que celle de David Hare quand il relève une phrase de Rumsfeld pour en faire le titre de sa pièce Stuff Happens. La visée de Hare est critique; pas celle de Vinaver (peut-être est-elle ironique, ou entend-elle simplement restituer sa surprise, son étonnement à la lecture de cette phrase et du terme « collapse »).

${ }^{40}$ Marie Darrieusecq, Rapport de police, P.O.L., 2010.

41 Ibid., p. 145.

42 Iphigénie Hôtel, in Théâtre populaire, $\mathrm{n}^{\mathrm{o}} 39,3^{\mathrm{e}}$ trim. 1960, p. 106. Cette déclaration est maintenue dans les éditions ultérieures de la pièce, sauf dans la dernière édition en date (Théâtre complet, vol. 2, Actes Sud, 2003), où n'est conservée que la mention du roman de Green. Cette mention, l'éditeur Gallimard (1963) l'avait même ajoutée sur la couverture et sur la page de garde (sous le titre) - peutêtre suite à la réaction de ce dernier (dont Michel Vinaver m'a dit qu'elle avait été plutôt froide), peutêtre aussi pour des raisons publicitaires (Gallimard étant l'éditeur français d'Henry Green).

${ }^{43}$ Voir Will Hammond, Dan Steward, Verbatim Verbatim: Techniques in Contemporary Documentary Theatre, Oberon, 2008. Stafford-Clark et Hare commencent l'un des entretiens en citant Einstein : savoir cacher ses sources serait le «secret of creativity » (définition classique de l'art); le théâtre verbatim fait précisément le contraire. 
pièce. Elle n'apparaît même pas sur le dernier état (qui est le tapuscrit de la traduction française, daté du 22 novembre 2001). Vinaver l'aurait-il ajoutée spécialement pour cette question de l'auctorialité, comme pour finir par une signature en bas du tableau ${ }^{44}$ ? Par ailleurs, on trouve, dans ce même tapuscrit, une première version de la « Note liminaire » :

[...] paroles rapportées, extraites < pour une large part> d'articles partus dans <tirées de $>$ la presse quotidienne. Traduetion française par l'auteur $<$ Version française par l'auteur $>$. La forme se rapproche de celle des cantates, des passions et des oratorios $<\mathrm{du} X V \mathrm{WH}^{\mathrm{e}}$ siècle $>\ldots{ }^{45}$

On sent que le positionnement de l'auteur par rapport à son texte n'est pas encore assuré (du fait de la grande originalité de cette pièce, aussi bien dans son propre corpus, que dans la production française contemporaine), qu'il cherche à formuler, mais aussi à comprendre. La partie française était d'abord appelée «traduction », puis "version», et enfin " adaptation » dans l'édition finale. Vinaver explique qu'il l'a réalisée en deux jours, pour une lecture de la pièce, devant un public français, fin 2001, mais l'enjeu était peut-être aussi de s'approprier définitivement l'œuvre (pour Nelson Goodman, une traduction, présentant par définition un autre texte, ne peut être qu'une autre œuvre). On remarque ainsi que Vinaver ne s'est pas servi des traductions françaises officielles, parues dans les journaux français, et l'on sait que l'exercice de la traduction a toujours été, pour lui, quelque chose de très important et relié à l'acte même d'écrire, dès ses tout premiers textes ${ }^{46}$. Enfin, il est intéressant de noter que les éditeurs de la pièce à l'étranger conservent la version américaine et mettent en regard la version dans la langue de leur pays (l'italien, l'espagnol). Selon Étienne Balibar (qui reprend des analyses de Benjamin), notre monde de la mondialisation n'est ni le monde de la «transparence réciproque, de la traductibilité intégrale», ni le monde de «l'incommunicabilité, de l'intraductibilité des cultures [...] Il est le monde des tentatives de traduction, qui laissent toujours un "reste" - mais ce reste est à son tour la condition du désir de communiquer ${ }^{47}$.»

\footnotetext{
${ }^{44}$ C'est même une signature stylistique : quand on est habitué à la lecture des textes de Vinaver, on remarque que cette réplique correspond plus nettement à son elocutio que toutes les autres de la pièce. On irait même jusqu'à affirmer qu'elle a d'abord été écrite en français, puis traduite en anglais.

${ }^{45}$ Michel Vinaver, tapuscrit de 11 septembre 2001, IMEC - VNV 35.8. On trouve ici un troisième avatar de l'expression « tiré de ». En rappelant les deux premiers, on obtient ainsi trois techniques ou principes littéraires très différents: Iphigénie Hôtel est une dérivation d'ordre hypertextuel (avec changement de « mode », du roman au théâtre - cf. Gérard Genette, Palimpsestes, Seuil, 1982), La fête chez le cordonnier est une traduction libre avec quelques effets proximisants, et 11 septembre 2001 un collage d'extraits de divers articles, paroles, discours réels.

${ }^{46}$ Ses nouvelles, comme «The Joker» (1944) ou «The Genesis of WM» (1947), il les a d'abord écrites en anglais, puis les a traduites en français. Pour «Le clos adoré » (1946), ce fut l'inverse, il l'a traduite en anglais («The Beloved Cottage »), et voilà ce qu'il en disait (Mémoire de fin d'études à Wesleyan University, 1947 - archives personnelles de l'auteur): «This translation was a specific assignement which I undertook against my wish. It proved to be an interesting, if sour, experience. Self-translation is an effective means for self-criticism, and it is the nearest literary equivalent to selfviolation. \)

${ }^{47}$ Etienne Balibar, « Une culture mondiale ? (1997), in Droit de cité, PUF Quadrige, 2002, p. 161.
} 


\section{Esthétique (philosophie de l'art) et poétique - l'à propos de}

\section{Les paradoxes de la mimèsis}

Avec 11 septembre 2001, Vinaver nous rappelle quelque chose de fondamental : que la composition est peut-être l'élément principal du fait littéraire (avant l'inventio ou l'elocutio). Pascal ne soutenait-il pas déjà la même chose ? «Qu'on ne dise pas que je n'ai rien dit de nouveau ; la disposition des matières est nouvelle $»^{48}$. La composition de 11 septembre 2001, on peut donc la reconstituer en trois étapes : recomposition de discours réels (nouvelles juxtapositions créées au sein d'une même réplique), collage de différents fragments hétérogènes (jusqu'au télescopage), et mise en page bilingue. La pièce dans son ensemble est, dit Vinaver, «l'invention (c'est là qu'elle intervient) d'un objet de parole en explosion » ${ }^{49}$.

Pour autant, tout n'est pas résolu. La grande question que pose Michel Vinaver à propos de 11 Septembre 2001 est celle de l'imitation. De très nombreuses notes (la plupart datant de 2005, au moment de la création américaine de la pièce par Cantarella) témoignent de cette réflexion : par un ultime retour à Aristote (comme un pied de nez aux théories du postdramatique), Vinaver affirme calmement que sa pièce est une imitation de l'événement ${ }^{50}$. Mais il ne définit jamais véritablement ce qu'il entend par là. Quand Vinaver décrit 11 septembre 2001 comme un «objet de parole en explosion", on a certes l'image d'une pièce qui, mimétiquement, représente l'explosion et l'effondrement des tours par la composition éclatée des paroles. Imitation ou analogie qui se précise quand il distingue l'imitation de la ressemblance : «L'imitation - n'est pas 'faire ressemblant' / (les portraitistes du pont NDame) $»^{51}$. Depuis la révolution conjointe de Cézanne et de la photographie, «faire ressemblant » est une ambition dévalorisée (sauf quand elle est radicalisée, chez les hyperréalistes par exemple ${ }^{52}$ ). Dévalorisée ou même exclue de fait du champ de l'art, inutilisable, dans la mesure où l'objet artistique est l'objet réel (Duchamp, Warhol...). En va-t-il ainsi des phrases de 11 septembre 2001 ? Une parole découpée et recollée avec d'autres, quelle trace garde-t-elle de sa réalité ? Qu'est-ce qui fait la propriété d'une parole, sa véracité ?

\footnotetext{
${ }^{48}$ C'est d'autant plus intéressant, dans le cas de Pascal, que l'ordre final de son recueil n'a pas été déterminé, et qu'il est ainsi devenu l'objet de toutes les rivalités scientifiques. Ce fragment par exemple, est classé VII, 9 dans l'édition Havet, porte le numéro 22 dans Brunschwicg, 747 dans Strowski, 65 dans Lafuma, 696 dans Chevalier, 1282 dans Kaplan...

${ }^{49}$ Michel Vinaver, «Mimèsis », dans le programme du spectacle mis en scène de Robert Cantarella, (on peut le trouver dans le dossier de presse du Théâtre de la Colline, 2006 :

http://www.colline.fr/telecharger.php?document=262).

${ }^{50}$ Citons quelques-unes des différentes mentions : Vinaver imite parce qu'un tel événement empêche l'imagination et il dit « réfléchir l'événement plutôt qu'y réfléchir » («Mimèsis », note déjà citée) ; le théâtre sert à «imiter la vie», ce qui est nécessaire «pour qu'on la comprenne » (note manuscrite, datée du 20 avril 2004, archives de l'auteur); "fixer l'événement dans son incandescence d'origine, fixer la surprise ou plutôt la reproduire, l'IMITER » (dans une liste lue à la Mousson d'été, reproduite dans Lexitextes $\mathrm{n}^{\circ}$ 9, L'Arche / Théâtre National de la Colline, p. 287) ; «Imiter l'événement (comme les artistes paléolithiques dans leurs cavernes) et pas du tout témoigner sur l'événement» (entretien avec Arnaud Meunier et Simon Chemama, in Le geste de témoigner, un dispositif pour le théâtre, Louvain-la-Neuve, Études théâtrales, parution fin 2011).

${ }^{51}$ Note manuscrite, sur laquelle figure aussi une citation de Proust, in dossier 11 septembre, archives personnelles de l'auteur.

52 Ou quand elle est recherchée pour semer le doute : comme les bouées en plastique suspendues de Jeff Koons, qui sont en fait en acier trempé (exposées à la Fondation Pinault dans une exposition qui était une célébration du « doute»).
} 
La tension vient du fait que Vinaver développe un discours très aristotélicien, avec la notion d'imitation et l'idée de faire un acte poétique (créer des imitations « fausses » ${ }^{53}$ ) bien plus qu'un travail d'historien, mais qu'il prend, en même temps, le pur réel comme matière de sa pièce (c'est l'image spéculaire, l'imitation «vraie» selon Platon; et l'Histoire selon Aristote). Dans 11 septembre 2001, serait ainsi défini un troisième genre : l'imitation vraie mais qui ne ressemble pas (ne ressemble pas parce que l'objet artistique est le collage de toutes les phrases réelles).

Qu'est-ce qu'une imitation? À quoi ce terme s'applique-t-il dans 11 septembre 2001 ? S'agit-il d'une classique imitation d'action ? On n'assiste pas à des représentations d'actions singulières en tout cas. Alors que l'approche de Dwyer et Flynn dans 102 minutes (qui rapportent les détails des solutions qu'ont trouvées les rescapés pour sortir du World Trade Center) est celle qui intéresse le plus Michel Vinaver ${ }^{54}$, la pièce ne montre aucune de ces actions, mais simplement des récits (cela aurait pu être intéressant, matériellement, corporellement, de les dramatiser davantage). Seule la révolte dans le dernier avion («Let's roll ») et l'épisode de l'ascenseur peuvent éventuellement donner lieu à une action mimétique (Arnaud Meunier a en partie travaillé dans ce sens). Quant à l'imitation d'une action, dans le sens aristotélicien général, elle serait bien difficile à identifier - elle nécessite sans doute les deux éléments dramaturgiques traditionnels que sont la causalité et les personnages, éléments absents de 11 septembre 2001. Ce passage de l'imitation d'une action à «l'imitation de l'événement » me semble important à relever.

Par ailleurs, cette imitation de l'événement est stylisée. Comme chez l'homme aux allumettes du film de Robert Cantarella sur Ground Zero ${ }^{55}$, il y a peut-être dans la conception de 11 septembre 2001 cette ambition de rendre compte d'un événement majeur avec des moyens minuscules. Vinaver conçoit une pièce de vingt pages, alors qu'il s'agit d'un des événements les plus importants des cinquante dernières années; pour figurer les explosions lors des crashs des avions, il dessine de petits traits sur son manuscrit (voir reproduction cidessus - ils apparaîtront sur tous les états manuscrits et tapuscrits, avant d'être supprimés pour l'édition de L'Arche), qui étaient ensuite représenté par l'auteur lors de ses lectures de la pièce avec Geoffrey Carey par le froissement d'une feuille de papier.

Ce dernier détail peut nous mettre sur une piste importante : Vinaver s'intéresse moins à la ressemblance qu'au support, et dès lors, paradoxalement, semble s'éloigner de la conception de l'art comme imitation. Danto fait cette remarque très pertinente : "La théorie mimétique de l'art doit son attrait moins à la notion de duplication qu'au fait qu'elle laisse entendre qu'une duplication adéquate peut transcender son médium ${ }^{56}$. Vinaver ne pense pas du tout l'imitation dans ce sens platonicien. 11 septembre 2001 ne transcende pas son médium, c'est-à-dire que la pièce n'oublie jamais qu'elle est une pièce, mais au contraire insiste làdessus. Le fait de devoir dire ou montrer les noms des personnages avant leur prise de parole, par exemple, revient même à exhiber la dramaticité de l'œuvre. Un théâtre de l'immanence comme celui de Vinaver est caractérisé, entre autres, par cette conscience du médium, en dehors de toute tentative d'illusionnisme intégrateur (du médium dans la duplication). Et

\footnotetext{
53 Cf. Danto, La transfiguration..., op. cit., p. 126-127.

54 Dwyer, Flynn, 102 minutes, op. cit. : «The immediate challenges these people faced were not geopolitical but intensely local : how, for instance, to open a jammed door, or navigate a flaming hallway ». Dans ses notes, Michel Vinaver fait souvent mention de ce livre.

55 Carrosserie, de Robert Cantarella, 2004 (http://www.robertcantarella.com/index.php?/film/carrosserie/). Voir notre entretien avec l'auteur dans ce même dossier (http://agon.ens-lyon.fr/index.php?id=1810).

${ }^{56}$ La transfiguration..., op. cit., p. 243-244.
} 
Robert Cantarella, dans l'entretien que nous avons eu avec lui, avait tout à fait raison de pointer la proximité avec le groupe Supports/Surface.

Les deux artistes auxquels Vinaver se réfère le plus souvent sont Rauschenberg et Dubuffet, qui sont de bons représentants de la conception de la peinture comme une activité pleinement matérielle: ils rendent visible l'acte de peindre lui-même. Danto montre que «l'acte de peindre était le motif et la substance des œuvres des années $1950{ }^{57}$, et que l'importance donnée au « coup de pinceau » était tel que « la question de savoir ce que l'artiste réalisait à travers lui ne se posait guère. Il était impossible de l'intégrer à une image, il était à part, il était ce qu'il était ${ }^{58}$. Des artistes comme eux, et Pollock, ou même Hantaï dont parle parfois Vinaver, «peignaient» bien plus qu'ils n'imitaient ${ }^{59}$. Le modernisme est un matérialisme.

Le geste de Vinaver, double, est le découpage et le collage - et ce geste, nous l'avons vu, est lui aussi mis sur le devant de la scène. Mais que peut-on appeler la matière (et le support) d'un texte - quelle est la «cause matérielle » d'un texte ? On dirait qu'il y a une ambiguïté, une confusion autour de cette question, déterminante pour l'ensemble de notre propos. Il est facile d'identifier la matière d'une œuvre plastique (en peinture ou sculpture il y a un rapport indispensable entre matière et forme), mais existe-t-il une matière des textes (et de la musique) ou n'est-elle pensable que par analogie ? Quand on parle de la matérialité d'un texte, on renvoie le plus souvent à ses sonorités, ce qui revient à prendre la facette la moins concrète et la moins stable de son être. On ne parle jamais de l'encre ou du papier, sans doute parce qu'un texte n'existe pas en un seul exemplaire; ce qu'on a entre les mains n'en est jamais qu'une représentation (ou occurrence, ou manifestation, selon les terminologies) - un texte est quelque chose de bien plus «idéal » qu'un tableau, et Platon ne l'a pas bien montré (ou plutôt ne pouvait pas, historiquement, le faire) ${ }^{60}$.

La question peut se poser en ces termes : cette matière peut-elle, d'un point de vue logique, être une phrase (c'est-à-dire ce qu'on appelle, depuis Benveniste, l'élément du « discours ») ? Ce qui paraît sûr, c'est qu'elle ne peut pas se confondre avec le « sens » (elle est seulement le substrat qui peut ensuite être formé). Passer d'un choix de mots (le travail poétique habituel) à un choix de phrases, comme le fait Vinaver dans 11 septembre 2001, serait un saut qui changerait radicalement la donne: on ne prend plus des sons qui appartiennent au grand stock du langage humain, mais des phrases déjà formées et sensées. Deux objections. D'une part, il y a toujours des cas limites : quand les phrases sont clairement utilisées pour leur sonorité, indépendamment de leur sens, comme dans les idéaux de poésie

\footnotetext{
57 Ibid., p. 99. Les années 50 à New York sont « l'apothéose de la matérialité de la peinture » (p. 84).

${ }^{58}$ Ibid., p. 180.

59 Dubuffet se distingue peut-être des autres, dans la mesure où il affirmait (mais sans doute paradoxalement) que, dans des séries comme celle des Texturologies, il imitait, copiait, de façon précise, des sols (ce qui, dans les faits, ne pouvait pas donner de tableaux très « figuratifs »).

60 D'une certaine façon, toute œuvre littéraire (à la différence d'un tableau par exemple) regagne, si l'on pense contre et avec Platon, des degrés de réalité. Elle redevient une sorte d' « idée », et l'existence par laquelle elle nous apparaît n'en est jamais qu'une exemplification (elle n'est pas détruite lorsqu'on détruit ses copies). D'autres théoriciens de l'art analysent le problème en utilisant la distinction peircienne entre type et occurrence. Genette, qui s'oppose à Danto sur ce point, rend compte très clairement de ces débats et se sert, lui, de la notion husserlienne d'idéalité, qui ne dénote pas la même abstraction (voir L'œuvre de l'art, op. cit., notamment p. 18 sq., 115 sq.). Si l'on reste sur le terrain du platonisme, une pièce de théâtre (mais c'est sans doute plus contestable encore) se déploirait sur trois niveaux: l'idée (la pièce), sa réalité matérielle (le texte imprimé, représentation première), et la représentation scénique (seconde).
} 
pure $^{61}$. Chez Vinaver c'est le cas du chorus « hijacked jets » ${ }^{62}$. D'autre part, même à propos des phrases « normales » (comme «I left the seventy-ninth floor with all my colleagues ») on peut penser, avec Antoine Compagnon, que le cheminement vers la citation (qui suit les étapes de la sollicitation, de l'accommodation, du soulignement et de l'ablation) «n'a rien à faire de la signification. La signification (sinon le sens) est la cinquième roue de ce carrosse $»^{63}$. Le véritable élément déclencheur, dans le travail de la citation, est ce qu'Antoine Compagnon appelle la «sollicitation", l'appel que lance le texte-source (même s'il ne précise jamais de quelle nature est cet appel). Cette idée de sollicitation permet de mieux comprendre la note liminaire française. Michel Vinaver avait traduit «words taken from daily newspapers » par «paroles provenant de la lecture de la presse quotidienne », ce qui n'est pas une traduction littérale : en utilisant le participe présent («tirées de » eût été plus proche de l'anglais) mais surtout en ajoutant «de la lecture », il insère un élément révélateur de l'essence même de la littérature. Écrire, c'est peut-être, avant tout, lire. Quand il l'exprime dans sa langue maternelle, les «paroles » ne sont plus simplement extraites des textes-sources, mais comme transformées par cette espèce de filtre qu'est la « lecture». Et celle-ci a à voir, continuerait Antoine Compagnon, avec une «passion » (« la citation tente de reproduire dans l'écriture une passion de lecture $\left.{ }^{64}\right)$. Or Vinaver dit non seulement citer les propos qui l'ont passionné, mais aussi citer l'événement lui-même (reçu comme un coup de foudre) sous forme d'une passion. Les «passions 》 de Bach étaient ses modèles musicaux premiers.

Enfin, même si l'on pense que toutes les phrases ne sont pas reprises uniquement à titre d'éléments matériels (11 septembre 2001 n'est pas un poème de Tzara avec phrases prises dans le journal au hasard, découpées, tirées au sort et recollées), leur statut devient complètement différent une fois qu'elles sont insérées dans la pièce. Ce n'est pas seulement dû aux modifications apportées, mais au simple fait de les déplacer à l'intérieur d'un nouveau système. Danto (analysant la reprise d'un schéma du critique d'art Erle Loran transformé en tableau par Lichtenstein) écrivait : «Le reproche de plagiat n'est donc pas justifié, puisque les deux objets appartiennent à des catégories différentes ${ }^{65}$. Attaquer l'auteur pour contrefaçon reviendrait à ne pas voir la transfiguration - comme un visiteur d'exposition naïf (et impudique) qui urinerait dans Fontaine de Duchamp (celui qui l'a fait ${ }^{66}$ était bien conscient de la transgression qu'un tel geste représentait : d'une certaine façon il prolongeait l'œuvre, et jouait avec le monde de l'art, comme Duchamp 80 ans plus tôt).

\footnotetext{
${ }^{61}$ Proust (« Combray », II) : «"La blanche Oloossone et la blanche Camyre” et "La fille de Minos et de Pasiphaé" » sont les deux vers qui, selon Bloch, ont «le mérite suprême de ne signifier absolument rien ». Mais il s'agissait là non pas de phrases, mais de vers (purement nominaux d'ailleurs).

${ }^{62} 11$ septembre 2001, p. 144. Voir aussi les scènes de fête de Par-dessus bord où se mêlent un très grand nombre de phrases tronquées et décontextualisées.

${ }^{63}$ Compagnon, op. cit., p. 26. Plus loin, il posera la question dans ces termes : «Un énoncé - une phrase, statement en anglais - n'est pas un signe. Mais l'objection vaut-elle encore pour une citation, pour un énoncé répété ? Est-ce qu'une phrase - sentence en anglais - ne peut pas, quand même, parfois, être un signe ? » (p. 58). Si, elle le peut ; en dégageant un «niveau d'analyse supérieur, celui des phénomènes interdiscursifs $»$.

${ }^{64}$ Ibid., p. 26. Plus loin, Compagnon dit même que «s'il y a une passion dans l'écriture », elle se présente sans guillemet (p. 42).

${ }^{65}$ La transfiguration..., op. cit., p. 230. Tout le travail de Danto dans ce livre consiste à dégager une philosophie de l'art à partir de ce genre d'œuvres contemporaines plus ou moins indiscernables d'objets « réels » (Boîte Brillo de Warholl par exemple).

${ }^{66}$ Pinoncelli, en 1993, au Carré d'Art de Nîmes.
} 
Le corollaire de cet usage d'un réel brut est le même chez Vinaver et chez les peintres américains des années 1950: il s'agit d'une forme d'idéologie anti-artistique (sans doute moins radicale que celle de Dada, mais tout aussi problématique). Ce qui tend à remplacer l'art est soit une pratique ordinaire (artisanale, qui fait fi de l'opposition entre faire et créer) soit extraordinaire (magique), mais en tout cas une pratique. Pourquoi Vinaver choisit-il l'exemple de Lascaux à chaque fois qu'il commente son geste d'imitateur? Il ajoute parfois qu'il existe de nombreuses interprétations de ces peintures rupestres, mais qu'on ne connaîtra jamais l'intention réelle de leurs auteurs. "Ils imitaient», voilà tout. Si Vinaver avait choisi, comme modèle, non pas Lascaux, mais, disons, les vases grecs antiques, les portraits hollandais du $\mathrm{XVI}^{\mathrm{e}}$ siècle ou encore les paysages impressionnistes français, le sens en eût été tout à fait différent. Il y a une dimension possiblement magique de la peinture préhistorique, comme tentative de recréation. Lascaux est peut-être l'équivalent de ce que Nietzsche déclarait au sujet des rites dionysiaques, à savoir qu'ils étaient la présentation magique du Dieu lui-même. « Avec le temps, écrit Danto, le fait de "tenir lieu de", donc la fonction dénotative, devenait de moins en moins importante pour les œuvres d'art, si l'on excepte les œuvres commémoratives, tels les portraits, les peintures d'histoire... ${ }^{67}$. Le philosophe américain présente ainsi l'évolution de l'art au fil du temps : c'était originellement « une partie de la réalité [...] avant d'en venir peu à peu à s'y opposer ${ }^{68}$, et c'est même en s'y opposant que l'art devient l'art. $\mathrm{Au} \mathrm{XX}^{\mathrm{e}}$ siècle, chez certains artistes, on retrouve la dimension première. Lascaux et mimèsis sont donc peut-être deux concepts qui ne collent pas, car une imitation suppose que celui qui crée l'objet comme celui qui le regarde sachent qu'il s'agit d'un simple artefact - ou seulement d'un artefact. En outre, les références que fait Vinaver à la musique confortent cette analyse, la musique étant l'art le moins mimétique (un morceau de musique est une expression plutôt qu'une représentation). Un bison sur une paroi, comme une fugue de Bach, ne sont pas à propos de quelque chose; ils sont.

Dans la philosophie de l'art de Danto, l'aboutness (l'à-propos-de) est un point crucial de différenciation entre l'œuvre et l'objet réel. «L'imitation devient une possibilité artistique à partir du moment où elle ne se borne plus à ressembler à quelque chose, comme le fait une image en miroir, mais est à propos de ce à quoi elle ressemble $»^{69}$. Or, Vinaver cherche précisément à éliminer l'about, l'à-propos-de ou le sur. Je n'écris pas sur le 11 septembre, ditil souvent ${ }^{70}$. Le problème est que Danto expliquait qu'un texte ne peut pas s'émanciper de ce trait relationnel : «Les mots peuvent posséder toutes les propriétés des entités du monde, sauf qu'ils sont à propos de lui et que lui est ce à propos de quoi ils sont, l'à-propos-de [aboutness] étant la propriété de différenciation cruciale », et il prévoit même la revendication : " Celui qui a soutenu qu'un poème ne devrait pas signifier mais être a énoncé une sorte d'incohérence ${ }^{71}$. Ce qui nous importe, déjà, est que Vinaver fasse ce genre de déclaration. « Je n'écris pas sur le

\footnotetext{
67 La transfiguration..., op. cit., p. 136. 11 septembre 2001 n'est ni une peinture d'histoire ni un portrait, mais peut-elle appartenir au groupe des « œuvres commémoratives »? Vinaver dit qu'il faut « fixer », " peut-être contre l'empâtement de la mémoire, contre ses aménagements » (« Mimèsis », texte cit.). Et le fait qu'elle ait été jouée pour l'anniversaire des dix ans des attentats le montre bien. Mais la remarque de Danto est assez surprenante : en quoi de telles œuvres « tiennent lieu de »?

${ }^{68}$ Ces deux dernières citations viennent de La transfiguration..., op. cit., p. 136.

${ }^{69}$ La transfiguration..., op. cit., p. 123-124.

${ }^{70} C f$. entretien avec Arnaud Meunier et Simon Chemama, in Le geste de témoigner, un dispositif pour le théâtre, Louvain-la-Neuve, Études théâtrales, parution fin 2011. De même, Vinaver pouvait dire qu'il n'écrivait pas « sur le travail » (Registres, numéro spécial nº1, hiver 2008-2009, p. 54).

${ }^{71}$ La transfiguration..., op. cit., respectivement p. 142 et 145.
} 
11 septembre » paraît signifier que Vinaver ne porte pas de jugement sur, qu'il ne fait que relever et constater. Comme dans cette note de l'auteur, qui donne une formulation encore plus forte (intransitive) :

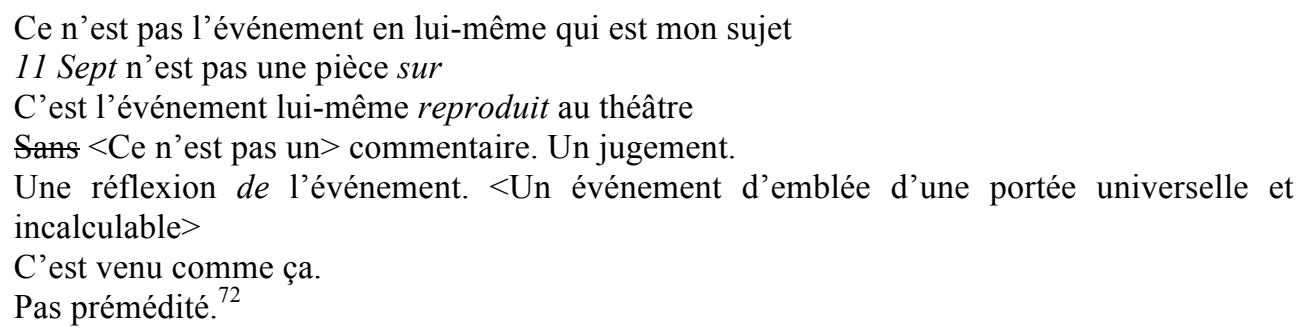

Mais « sur », en français, est une proposition peu sûre. Ce n'est pas seulement «about », c'est aussi «on ». Écrire sur, comme s'il y avait déjà une feuille, celle de toutes les paroles émises le 11 septembre ou justement à propos du 11 septembre, et que Vinaver ne voulait pas en ajouter (l'image du palimpseste). D'une certaine façon, c'est la troisième étape dans son évolution vers un retrait de l'énonciation personnelle, après le passage du roman au théâtre (disparition du narrateur) au milieu des années 1950.

Notre thèse est celle-ci : la conjonction de l'usage du réel brut et de l'affirmation d'une non-aboutness (une pratique et une attitude qui sont traditionnellement non-artistiques) donne en fait la défense la plus forte du geste artistique. Michel Vinaver réalise en littérature ce qu'un artiste comme Rauschenberg a fait en art plastique.

Vinaver crée un théâtre différent du verbatim qui fait bien, lui aussi, usage du réel brut, mais qui en revanche affirme clairement sur/contre quoi sont les pièces (voir par exemple Guantanamo de Brittain et Slovo). L'idée qui est la plus souvent exprimée à propos du théâtre verbatim, dans son rapport au réel, est que l'imitation dramatique viendrait suppléer au journalisme contemporain déclinant. Ce n'est pas un théâtre puisant dans le journalisme, mais plutôt du journalisme qui monte sur scène. « This sort of theatre provides what journalism fails to provide, and at a time when it is sorely needed ${ }^{73}$. C'est aussi ce que dit l'auteur David Edgar dans un entretien ${ }^{74}$. De nombreuses pièces sont en effet exclusivement composées d'extraits d'entretiens, souvent réalisés par l'auteur lui-même (comme Life After Scandal de Robin Soans ${ }^{75}$ ) ou même avec l'aide des acteurs (dans The Permanent Way de David Hare par exemple $^{76}$ ). Inversement, Vinaver construit un théâtre qui diffère aussi des courants type

\footnotetext{
72 Michel Vinaver, note non datée mais apparemment destinée au journal belge Le Temps. Archives personnelles de l'auteur.

73 Will Hammond, Dan Steward, Verbatim Verbatim, op. cit., «Introduction », p. 10. Dans l'entretien de ce même ouvrage que nous avons cité, on peut lire aussi cette phrase : "Verbatim does what journalism fails to do » (p. 62).

74 Dans The Guardian du 27 septembre 2008 (http://www.guardian.co.uk/stage/2008/sep/27/theatre.davidedgar) : « Why has this decade seen the rise of a vibrant theatre of reportage? Playwright David Edgar points to a decline in conventional journalism and TV documentary ». Des propos très semblables de ce même auteur sont aussi rapportés par Clare Finburgh, dans «Le théâtre britannique contemporain post-11 septembre 2001, du théâtre verbatim à l'allégorie », in D. Lescot et L. Véray (dir.), Les Mises en scène de la guerre au XXe siècle. Théâtre et cinéma, Paris, Nouveau monde éditions, 2011, p. 587.

75 Robin Soans, Life After Scandal, 2007. Dans ses remerciements par exemple l'auteur remercie « all the contributors for their generous and courageous agreement to talk on a difficult subject». Ce qui est original au début d'une pièce de théâtre.

76 David Hare, The Permanent Way, faber \& faber, 2003. Cette pièce porte sur le système ferroviaire anglais, à l'occasion d'un très grave accident de train. Dans ces cas, d'ailleurs, la question de l'autorité
} 
Nouveau Roman (ou de l'Absurde) : ils affirmaient aussi ne pas écrire sur quelque chose, mais inventaient le contenu des œuvres. On peut remarquer que ces courants tentaient parfois d'écrire sur rien, ce qui n'est d'ailleurs pas du tout la négation d'un à-propos-de mais sa version la plus paradoxale.

Pourquoi considérer ce positionnement comme la «défense la plus forte du geste artistique », alors qu'il est le plus éloigné de l'art «classique », traditionnel (celui qui n'est plus très souvent considéré comme geste) ? Peut-être parce qu'il en dégage la quintessence (ce qui reste après distillation totale), à savoir la composition, et un certain mode de présentation du contenu, mettant en valeur le médium. Serait-ce pour cette raison que Vinaver a arrêté d'écrire: la reconnaissance que 11 septembre 2001 était un aboutissement magistral, la destination de son œuvre, dont Les Coréens (1956) marquaient la première étape, Par-dessus bord la seconde, King (1998) la troisième ?

Dans 11 septembre 2001, Vinaver montre la grande attention qu'il porte à la parole. S'il ne crée pas beaucoup d' " actions » physiques, c'est que la parole doit agir. Vinaver a visé dans cette pièce l'imitation de l'événement par les paroles ${ }^{77}$. Dans la note qui suivait Iphigénie Hôtel, Vinaver imaginait déjà ce qui «pourrait amener le théâtre à se délier »: "attacher moins d'importance [...] à ce qui est dit, davantage au fait que ce qui est dit est dit $»^{78}$. Dans 11 septembre 2001, en passant souvent du discours indirect des articles-sources au discours direct des répliques, il défait le travail des journalistes de renvoi à un sens, pour réintroduire seulement une dénotation. Nous faisons allusion aux théories de Frege - dans une citation au style direct, « les mots prononcés dénotent les mots d'autrui et ce sont ces derniers qui ont la dénotation habituelle»; dans le cas du discours indirect, « on parle du sens des paroles d'un autre $»^{79}$. La seule anomalie, par rapport à la modélisation logique de Frege, est que Vinaver n'utilise pas de guillemets. Mais c'est peut-être ainsi que Vinaver imite radicalement (à la racine), et qu'il reproduit l'événement. C'est peut-être ainsi qu'il revient au niveau premier de l'énonciation, et d'une certaine façon nie la citation (et nie aussi son propre statut de sujet, pour ne plus s'attribuer qu'un rôle technique). En recréant un dialogue, Vinaver défait des citations plus qu'il n'en fait (ce qui apparaît comme un hommage infini rendu aux paroles et aux personnes qui en furent les émetteurs). C'est d'autant plus vrai qu'il n'y a dans la pièce que des phrases prélevées, et pas de discours auctorial qui viendrait les unir (la dernière réplique, inventée, ne peut pas être tenue pour telle).

\section{Du poétique au politique}

Que la forme exprime in fine quelque chose au sujet du contenu, voilà ce qui constitue, selon Danto, le texte en œuvre d'art. Il ne faut pas avoir peur d'interpréter, de chercher à lire les faits stylistiques et rhétoriques d'un texte (les choix de conserver tel ou tel passage d'un article, de remplacer tel mot, de passer à la ligne à tel endroit...). Sans affirmer $l a$ vérité définitive du texte, on doit défendre l'interprétation que l'on estime, à un moment

ne se pose plus, car si aucun des mots n'est inventé par les auteurs, ce sont ces derniers qui les ont commandés.

77 Plusieurs articles soutiennent que cette pièce est avant tout une représentation de représentations. Cela nous semble difficile à concilier avec l'idée que Vinaver défend, d'une imitation radicale du choc et de l'événement qu'il a simplement reproduit. Nous préférons donc nous en tenir à cette idée d'imitation des paroles.

78 Iphigénie Hôtel, version du Théâtre Populaire, op. cit., p. 106.

${ }^{79}$ Frege, « Sens et dénotation » (1892), cité par Antoine Compagnon, op. cit., p. 85. 
donné (dans un contexte culturel et politique donné) être la meilleure. Vinaver reconnaissait d'ailleurs, dans son très beau texte sur «l'adaptation», que l'interprétation était le prolongement normal de l'écriture : il retraçait la chaîne des adaptations de la Fête $d u$ cordonnier : chronique de Stowe (XVI ${ }^{\mathrm{e}}$ s.), récit de Deloney (1597), pièce de Dekker (1599), pièce de Vinaver (1959), et adaptation du spectateur (" chaîne » qui ressemble, si l'on veut, aux séries infinies d'« interprétants» peirciens). «Nous abolissons partiellement, écrivait Vinaver, leurs intentions originelles et nous leur substituons les nôtres. Continuellement, nous adaptons ${ }^{80}$.

L'étude génétique permet d'imaginer, de façon assez concrète, ce que les textes auraient pu être, et aide ainsi à mieux comprendre ce qu'ils sont. Elle montre, dans la plupart des cas, qu'il y a toujours un choix, que ce n'est jamais le seul hasard qui décide. Au moins deux passages travaillés dans les brouillons de 11 septembre 2001 n'ont finalement pas été retenus dans la version éditée (ils disparaissent dès la première version manuscrite). Il s'agissait de deux interviews, faites par le «journaliste», que Michel Vinaver adapta sur le même modèle que les autres, mais qu'il n'essaya pas, ensuite, d'insérer dans le montage de la pièce. L'une convoquait un producteur de cinéma, le directeur d'édition de Farrar, Straus \& Giroux et un créateur de sitcom comique de $\mathrm{NBC}^{81}$. Ces trois personnes commentaient les conséquences des attentats du 11 septembre sur l'industrie du divertissement: les bouleversements qui étaient en train de se produire sur les thèmes des œuvres produites et dans l'esprit du public (qui semblait ne plus accepter d'œuvres frivoles, mais pas non plus d'œuvres trop directement liées à l'attaque des tours). La deuxième interview regroupait un ingénieur, un professeur de physique, un des constructeurs des Twin Towers, et enfin le patron d'une entreprise de démolition, invités à expliquer, d'un point de vue purement matériel, la chute des tours $^{82}$. Voilà l'adaptation qu'en avait réalisée Michel Vinaver :

\section{DEMOLITION ENGINEER}

As each concrete floor in a falling building pancakes into the one below it

Their combined weight grows so large that they and everything in their path

Break into pieces

And the pieces are pounded smaller and smaller until mostly dust and small rubble remain JOURN

Frank Moscatelli you are a professor of physics at Swarthmore College in Pennsylvania you calculated that the total energy released in the disaster was one-twentieth of the atom bomb dropped on Hiroshima

\section{MOSCATELLI}

And most of this energy was supplied by gravitational collapse

People don't think of an item sitting still as having energy

But it does

JOURN

Jon Magnusson you are chairman of Skilling Ward Magnusson Barkshire a structural engineering company that worked out the original World Trade Center design

MAGNUSSON

The $<$ No question that the $>$ enormous energy released in the collapse was enough to pulverize the 110-story towers

And their contents

JOURN

\footnotetext{
${ }^{80}$ Michel Vinaver, « De l'adaptation », art. cit., p. 84. Il donne comme autre exemple la succession de transformations (physiques et interprétatives) subies par une main en bronze d'origine siamoise.

81 Vinaver était parti de l'article de John Leland and Peter Marks, « A Nation Challenged: Culture; New Look for Entertainment In a Terror-Conscious World», The New York Times, 24 septembre 2001.

82 À partir de l'article de Warren E. Leary, «The Physics of Turning a Tower Into a Cloud of Dust and Rubble », International Herald Tribune, 27 septembre 2001.
} 
James Redyke you are president of Dykon Blasting a demolition company

REDYKE

A building is like an accordion

When it collapses the air has to exit someplace

MAGNUSSON

We were surprised however

Initially

That the buildings went straight down

Without sliding to the side or toppling

Mais seul le titre de l'article-source sera conservé dans la pièce, dans un "chorus » d'une seule ligne : "The Physics of Turning a Tower into a Cloud of Dust and Rubble » (p. 166).

Au-delà du plaisir archéologique que procurent ces découvertes, on peut se demander pourquoi ces deux passages n'ont pas trouvé leur place dans la pièce. Or il semble que la réponse tienne au statut des personnes interrogées. Celles que Michel Vinaver a choisi de faire apparaître (et qui deviennent ainsi des personnages) sont toutes impliquées dans l'action : elles en sont les victimes (Madeline, Todd...), les auteurs (Ben Laden), les auteurs et victimes (Atta), les rescapés (qui forment le plus gros des troupes, puisque ce sont eux qui peuvent témoigner), et enfin celles qui doivent en tirer des conséquences politiques directes (Bush, Rumsfeld). Les personnes écartées, elles, n'étaient que des commentateurs et c'est ce statut qui semble les disqualifier. De plus, les ingénieurs montrent une confiance un peu ridicule dans le pouvoir de la raison - ils disent comprendre parfaitement ce qui s'est passé ${ }^{83}$; quant aux professionnels de la «culture », leurs analyses sont forcément biaisées par la proximité avec l'événement.

Cependant, il existe bien dans la pièce (en dehors du chœur et du journaliste, qui ont des fonctions dramaturgiques très particulières) un petit groupe de personnages commentateurs et extérieurs à l'action. Ce sont les deux traders, qui parlent du délai nécessaire avant la réouverture des " financial markets ${ }^{84}$. Mais on remarque une chose : leur identité et leur énonciation diffèrent de celles des autres personnages. Vinaver ne donne pas leur nom (alors qu'il le fait pour toutes les autres personnes citées à partir d'interviews, et que ces personnages sont bien identifiés dans l'article-source ${ }^{85}$ ); les personnes citées n'étaient d'ailleurs pas spécifiquement des «traders », mais des analystes financiers et un professeur d'économie $^{86}$ (déplacement qui, lui aussi, constitue une exception); et enfin, Vinaver ne conclut pas ce passage sur la même tonalité que l'article (qui terminait par cette phrase de l'un des analystes : "Obviously, emotionally we will never recover from the loss of family and friends »). Nous pensons qu'il y a là un fait stylistique marquant, que nous ne pouvons pas ne pas interpréter.

De quoi le 11 septembre 2001 est-il la date ? D'un attentat terrible assurément, mais aussi de l'épisode d'un « conflit ». Dans une note en 14 points, Michel Vinaver écrivait :

\footnotetext{
${ }^{83}$ Une brèche dans leurs certitudes semble s'ouvrir, à la fin de ce passsage, avec le «we were surprised», mais elle est vite comblée par l'adverbe «initially », mis en valeur puisqu'il occupe une ligne à lui tout seul. C'est d'autant plus marqué, et ironique, que la formulation dans l'article-source était différente (avec antéposition habituelle de l'adverbe) : «Mr. Magnusson and others said they were initially surprised that... ».

${ }^{84} 11$ septembre 2001, p. 164-165 (leurs propos sont entrelacés avec ceux d'Atta) - juste avant le chorus déjà cité, titre de l'article sur les commentaires des ingénieurs.

${ }^{85}$ Mitchell Martin, «Trading Restart Likely to Be Choppy », The New York Times, 14 septembre 2001 (http://www.nytimes.com/2001/09/14/news/14iht-shutdown_ed3_html)

${ }^{86}$ Ils sont trois en effet dans l'article-source ; l'un est « chief market analyst at Jefferies \& Co. », l'autre «professor at the Georgetown University Business School», et le dernier « chief economist of High Frequency Economics Ltd. ».
} 
6. Qui est le plus terroriste des deux ? L'occident n'a pas cessé de semer la terreur dans le monde. Colonialisme. Indignation qui ne fait pas un pli à l'égard du terrorisme en tant que tel, alors que nous nous sommes les civilisés. C'est contaminant. Il faut de temps en temps se cogner, se gratter.

Une telle remarque, dans le «camp » occidental, est très rare; elle est généralement le fait de sympathisants islamistes ou d'intellectuels provocateurs (catégories auxquelles n'appartient pas Vinaver). Clare Finburgh, dans un article sur le théâtre britannique contemporain, montrait en effet comment la grande majorité des discours sur le 11 septembre "ont résulté d'une évacuation des contextes historiques et politiques, et d'une fabrication de fictions idéologiques ${ }^{87}$. Les deux notes qui entourent ce propos aident à mieux comprendre la position de Vinaver :

5. La force du faible et la faiblesse du fort.

7. Le symbole World trade. Ce que j'ai appris, c'est que dans les tours jumelles, il n'y avait plus de World trade. Les spécialistes du commerce mondial, pour lesquels les deux tours avaient été construites..., il n'y avait plus une seule entreprise dans cette spécialité. Remplacées par des sociétés purement financières.

La première, de par son caractère de sentence générale, donne une résonnance quasi mythique (ou biblique) à l'événement, le désactualise ${ }^{88}$. La suivante, au contraire, recontextualise l'événement dans le capitalisme contemporain. Le capitalisme pour lequel a travaillé Vinaver était différent : capital, fabrication, commerce, profits. Il est devenu (depuis la fin des années 1980) excessivement financiarisé, et s'est transformé. Il s'est virtualisé, et n'a consisté qu'en un marché de l'argent.

Si 11 septembre 2001 n'est pas une pièce critique, elle donne en tout cas un espace pour la critique. Dire de cette pièce qu'elle est consensuelle (« la version la plus sophistiquée des pièces consensuelles ${ }^{89}$ ), c'est ne pas voir cet espace important ouvert par d'infimes choix de composition. 11 septembre 2001 s'ouvre sur critique du capitalisme dématérialisé (financiarisé) et dérégulé. Le trader en est la figure (pas le responsable unique), qui « joue » avec le monde (Bush ni Ben Laden ne jouaient), qui spécule, indifféremment, sur les monnaies ou les céréales, et, armé de nouvelles techniques mathématiques et de forts moyens de pression sur les États, ne peut même plus vraiment perdre.

Je pense que la démarche poétique de Vinaver colle à cette critique possible. Il invente une écriture matérielle (à la fois travail artisanal et fondation matérialiste), contre la dématérialisation du pouvoir et du capitalisme; une écriture de détournement des textes, de vol peut-être ${ }^{90}$, ou de partage, qui est profondément liée au sujet de la pièce. Alors que dans le théâtre verbatim, le prélèvement de discours existants est une "technique » applicable à

${ }^{87}$ Clare Finburgh, « Le théâtre britannique contemporain... », art. cit., p. 584.

${ }^{88}$ Vinaver dit souvent avoir été frappé par la dimension immédiatement mythique de l'événement. Il a d'ailleurs, juste après avoir écrit 11 septembre 2001, adapté Les Troyennes d'Euripide (créée avec 11 septembre 2001, par Domenech Reixach et Ramon Simo au Théâtre national de Catalogne en 2002).

${ }^{89}$ Olivier Neveux, «Présences critiques de l'impérialisme dans le théâtre contemporain », Les Mises en scène de la guerre au $X X^{e}$ siècle, op. cit., p. 514.

90 Était-ce dans le sens de «voler» que Planchon utilisait le terme «piquer», en 1973, à propos de Par-dessus bord qu'il venait de créer? Il disait que Vinaver était le seul auteur à savoir " "piquer" cette espèce de discours confus et en même temps très savant, qui passe dans certaines publications que tout le monde lit, et qui constitue la matière des grands journaux, la matière des grands hebdomadaires, etc... Or, il y a là quelque chose sur le plan littéraire qui est exemplaire et très étonnant. » 
l'infini, Vinaver a radicalisé une des tendances de son écriture (déjà décelable dans Portrait d'une femme) pour cette pièce-là.

Nous avions mis en exergue une citation amusante qui évoquait la pratique " postmoderne » de la citation chez Godard. Après avoir vu Tout va bien, le film de Godard sorti en 1972, Michel Vinaver écrivait :

Volontariste. Se casse la gueule à essayer de faire quelque chose. Une œuvre doit d'abord être, pour devenir agissante.

Veut être objectif. Est tout le contraire. Subjectif à mort et dévoile la solitude du réalisateur. Film sans communication / transmission / chaleur. ${ }^{91}$

Il nous semble que 11 septembre 2001 a réussi l'inverse: pièce politique, mais dont la politisation est à trouver dans la matérialité même, dans son travail de la copie, du collage et de la variation.

$\underline{\text { Pour citer ce document : }}$

Simon Chemama, «Recopiage et imitation », Agôn [En ligne], Dossiers, HS n ${ }^{\circ} 1$ : Mettre en scène l'événement, 11 septembre 2001 de Michel Vinaver, mis à jour le : 27/10/2011, URL : http://agon.ens-lyon.fr/index.php?id=1810.

\footnotetext{
${ }^{91}$ Note manuscrite, IMEC - VNV 14.6 (dossier La demande d'emploi). Le film de Godard montre une grève dans une usine, avec séquestration du patron.
} 